\title{
EFFECT OF CAFFEINE INGESTION ON HIGH-INTENSITY INTERMITTENT EXERCISE PERFORMANCE IN ATHLETES
}

\author{
A Thesis Submitted to the College of \\ Graduate Studies and Research \\ in Partial Fulfillment of the Requirements \\ for the Degree of Master of Science \\ in the College of Kinesiology \\ University of Saskatchewan \\ Saskatoon
}

By

Heather Kim Whelan

Spring 1999

(C) Copyright Heather K. Whelan, 1999. All rights reserved. 


\title{
PERMISSION TO USE
}

In presenting this thesis in partial fulfillment of the requirements for a Postgraduate degree from the University of Saskatchewan, I agree that the Libraries of this University may make it freely available for inspection. I further agree that permission for copying of this thesis in any manner, in whole or in part, for scholarly purposes may be granted by the professor or professors who supervised my thesis work or, in their absence, by the Head of the Department or the Dean of the College in which my thesis work was done. It is understood that any copying or publication or use of this thesis or parts thereof for financial gain shall not be allowed without my written permission. It is also understood that due recognition shall be given to me and to the University of Saskatchewan in any scholarly use which may be made of any material in my thesis.

Requests for permission to copy or to make other use of material in this thesis in whole or part should be addressed to:

\author{
Dean of the College of Kinesiology \\ University of Saskatchewan \\ 105 Gymnasium Place \\ Saskatoon, Saskatchewan
}

$\mathrm{S} 7 \mathrm{~N} 5 \mathrm{C} 2$ 


\begin{abstract}
To determine whether acute caffeine ingestion improves high-intensity intermittent exercise performance, 17 college-level, competitive athletes ( 9 males, 8 females, mean age 22 years) performed caffeine and placebo trials in a double-blind randomized study. Caffeine and placebo (lactose) doses of $6 \mathrm{mg} / \mathrm{kg}$ body weight
\end{abstract} (rounded to nearest $100 \mathrm{mg}$ ) were given in $100 \mathrm{mg}$ capsules; a dose consistently shown to produce urine caffeine levels below the maximum permitted by the International Olympic Committee. Habitual caffeine consumption, usual physical activity level, and food intake for 3 days prior to each trial were determined by questionnaire. At each trial, one week apart, subjects performed three 30 second sprints on a treadmill ( $7.5 \mathrm{mph}, 20 \%$ incline) separated by 2 minutes of walking, followed by a final sprint to exhaustion. Time to exhaustion (TM-EXH) was recorded and ranged from 20-90 seconds. Subjects reported a rating of perceived exertion (RPE) at the end of each sprint. Fingertip blood lactate (LACT) was measured following the third 30 second sprint, final sprint, and after 5 minutes of recovery. Respiratory variables were measured throughout the trial and the respiratory exchange ratio (RER) was determined for each sprint and recovery period within each trial. Repeated measures ANOVA showed no significant differences between the caffeine and placebo trials for TM-EXH, RPE, LACT, or RER. However, although not significant, there was an average increase in TM-EXH during the caffeine trial by 2 seconds (4\%); with 6 of 9 males and 5 of 8 females running longer during the caffeine trial. Habitual caffeine consumption did not appear to influence the results. In conclusion, the ingestion of caffeine prior to high intensity intermittent exercise seems to improve performance by a narrow and statistically non-significant margin. 


\section{ACKNOWLEDGEMENTS}

I would like to thank my advisor, Dr. Don Drinkwater and committee members, Dr. Dick Bell, Dr. Phil Chilibeck, and Dr. Kevin Spink for their input towards this thesis. Their time, support, patience, and help were greatly appreciated throughout this project. Also, thank you to Candace Schacter for agreeing to be my external examiner.

The Dean's Research Fund of the College of Kinesiology at the University of Saskatchewan funded this project. The financial support was very much appreciated. Thank you to the many individuals who were directly involved in collecting the data for this project. Doug Jacobson monitored the double blind key and was a huge help with all of the laboratory equipment. Many of my fellow students volunteered many hours in the lab testing the participants. This project would not have been possible without all of these people.

Finally, I need to thank my mother Barbara, sister Wendy, brother Tim, and my extended family for their continual support and encouragement. Everything is so much easier when you have so many people who believe in you. 


\section{DEDICATION}

This thesis is dedicated to my family: my father Christopher (1948-1984), my mother Barbara, my sister Wendy, and my brother Timothy. I remember most my Dad's sense of humor and how he could always cheer me up. Mum has always provided love and encouragement for whatever I decided to do. She is always there cheering me on. Wendy taught me to strive for excellence in everything I do by doing so herself. Tim has always known how to make me laugh and he taught me how to laugh at myself and see the funny side of any situation.

"So much of what is best in us is bound up in our love of family, that it remains the measure of our stability because it measures our sense of loyalty. All other pacts of love or fear derive from it and are modeled upon it."

Haniel Long 


\section{TABLE OF CONTENTS}

PERMISSION TO USE .................................................................

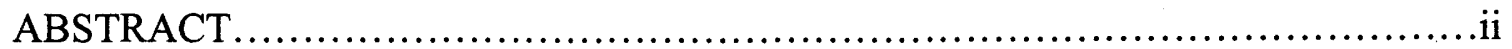

ACKNOWLEDGEMENTS .......................................................

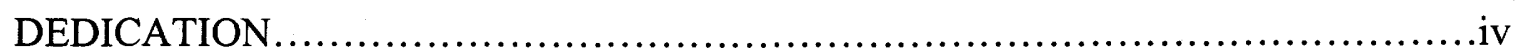

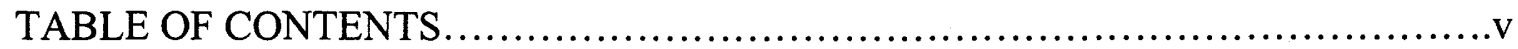

LIST OF TABLES .......................................................................

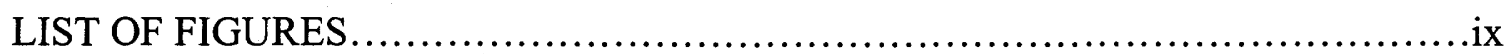

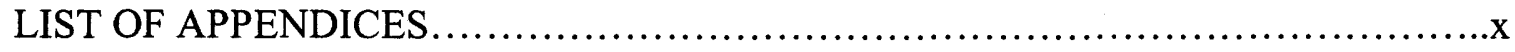

CHAPTER 1 SCIENTIFIC FRAMEWORK .........................................

$1.1 \quad$ Introduction............................................................

$1.2 \quad$ Review of Literature.................................................

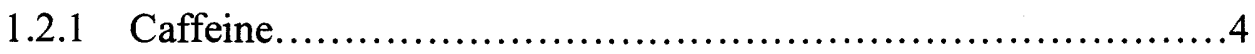

1.2.2 Potential Mechanisms for the Ergogenic Effect of Caffeine.........6

1.2.3 Caffeine and Endurance Exercise..............................10

1.2.4 Caffeine and High Intensity Continuous Exercise.................11

1.2.5 Caffeine and High Intensity Intermittent Exercise...............15

1.2.6 Caffeine and Perceived Exertion during Exercise.................17

1.2.7 Caffeine and Blood Lactate during Exercise....................18

1.2.8 Caffeine and Respiratory Factors during Exercise...............20

1.2.9 Caffeine Dose................................................22

1.2.10 The Effect of Habitual Caffeine Consumption....................23 
1.3 Statement of the Problem and Hypotheses..............................26

1.3.1 Statement of the Problem.........................................26

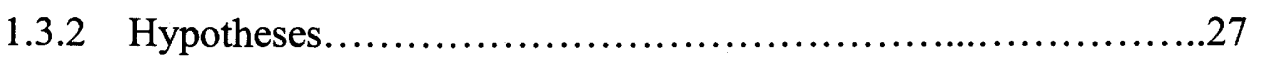

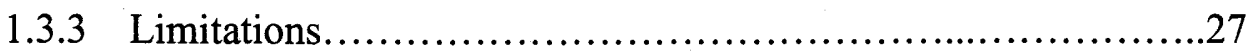

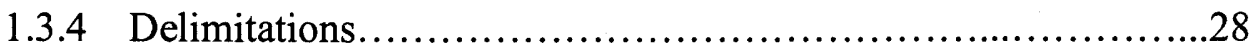

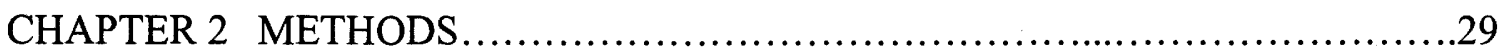

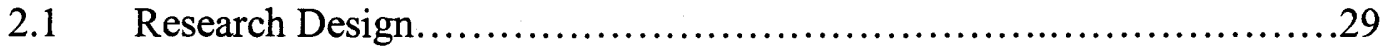

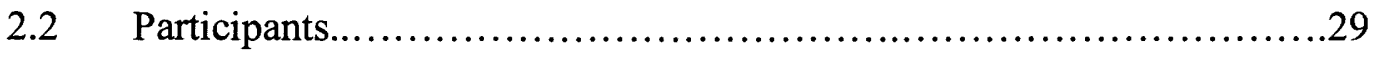

$2.3 \quad$ Procedures................................................................

2.3.1 Test Protocol................................................... 31

2.3.2 Physical Activity Measurement..................................33

2.3.3 Habitual Caffeine Consumption Measurement.....................33

2.3.4 Dietary Assessment..............................................

2.3.5 Treadmill Accustomization..........................................34

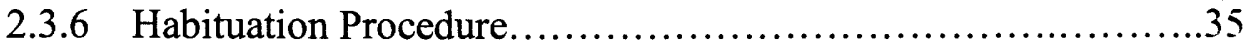

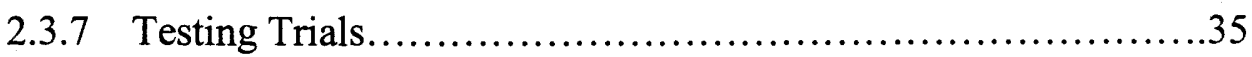

2.3.8 Capsule Administration......................................40

$2.4 \quad$ Statistical Analyses.................................................40

CHAPTER 3 RESULTS AND DISCUSSION ..................................42

$3.1 \quad$ Results................................................................42

3.1.1 Participant Characteristics...................................43

3.1.2 Time to Exhaustion............................................43

3.1.3 Rating of Perceived Exertion.................................48 
3.1.4 Blood Lactate................................................48

3.1.5 Respiratory Factors...........................................49

3.1.6 Heart Rate...................................................

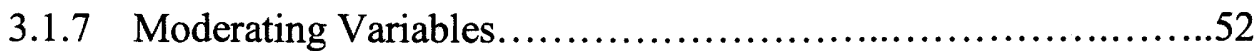

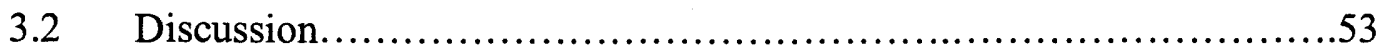

CHAPTER 4 SUMMARY AND CONCLUSIONS.................................63

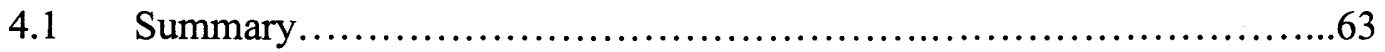

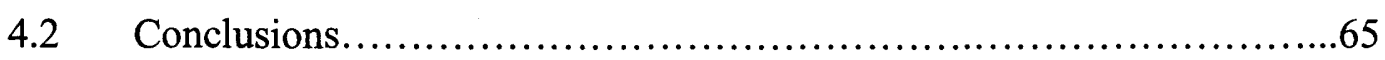

4.3 Recommendations for Future Research..............................66

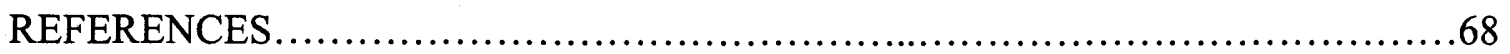

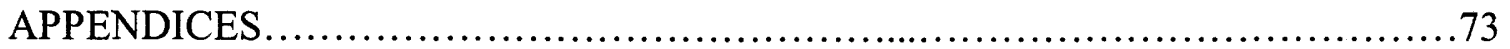




\section{LIST OF TABLES}

TABLE 2.1 Participant descriptive characteristics (mean \pm standard deviation)........31

TABLE 3.1 Pearson correlations for seventeen participants during the placebo trial...45

TABLE 3.2 Pearson correlations for seventeen participants during the caffeine trial..46

TABLE 3.3 Values of rating of perceived exertion (mean \pm standard deviation) during the experimental trials for seventeen subjects......................48

TABLE 3.4 Blood lactate values (mean \pm standard deviation) during the experimental trials for seventeen subjects.

TABLE 3.5 Respiratory exchange ratio values (mean \pm standard deviation) during the experimental trials for seventeen subjects.

TABLE 3.6 Ventilation volume values (mean \pm standard deviation) during the experimental trials for seventeen subjects

TABLE 3.7 Heart rate values (mean \pm standard deviation) during the experimental trials for seventeen subjects.

TABLE 3.8 Dietary variables (mean \pm standard deviation) for the three days prior to the experimental trail for seventeen participants. 


\section{LIST OF FIGURES}

FIGURE 3.1 Mean time to exhaustion ( $\pm 95 \%$ standard error of the mean) during the experimental trials in seventeen male and female subjects...............44

FIGURE 3.2 Individual time to exhaustion values during the experimental trials in eight female subjects......................................................47

FIGURE 3.3 Individual time to exhaustion values during the experimental trials in nine male subjects....................................................... 


\section{LIST OF APPENDICES}

APPENDIX A

APPENDIX B

APPENDIX C

APPENDIX D

APPENDIX E

APPENDIX F

APPENDIX G

APPENDIX $\mathrm{H}$

APPENDIX I

APPENDIX $\mathrm{J}$

APPENDIX K

APPENDIX L

APPENDIX M
Consent Form.

Three-Day Food Record. 77

Subject Instruction Form. .85

Data Recording Sheet. .87

Leisure Time Exercise Questionnaire. .89

Estimation of Habitual Caffeine Consumption Questionnaire.....91

Diet Analysis Print-Out........................................

PAR-Q (Physical Activity Readiness Questionnaire).............98

Background Information Form...............................100

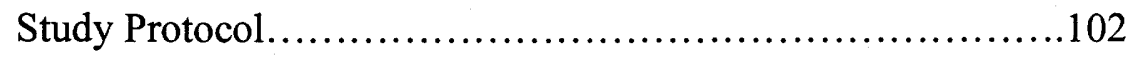

Metabolic Cart Print-Out. .108

Borg Scale of Rating of Perceived Exertion...................112

Blood Lactate Analysis Print-Out.

114 


\section{CHAPTER 1}

\section{SCIENTIFIC FRAMEWORK}

\subsection{Introduction}

In the world of athletics, a philosophy to win at all costs has developed. Professional athletes are making millions of dollars per year and Olympic Games medallists are returning home to huge monetary gains through endorsements and advertising (Haynes \& Fitch, 1992). At elite levels of competition, where the difference between the winner and the loser is often hundredths of seconds, one goal, or one point, athletes often search for the little things that might give them an edge over their opponents, including a wide variety of actions and products collectively called ergogenic aids.

An ergogenic aid is a substance, product, action, or tool that increases work capacity or improves performance or recovery from performance (Williams, 1995a). These can range from mechanical aids such as lightweight equipment to pharmacological aids such as anabolic steroids that can have serious side effects. Ergogenic aids also include psychological aids like hypnosis and physiological aids like blood doping (Williams, 1995a). To prevent the widespread use of ergogenic aids, some of which can have dangerous consequences, the International Olympic Committee (IOC) has 
developed a list of banned and restricted products (Haynes \& Fitch, 1992). The IOC updates this list every year. Athletes are always trying to stay one step ahead of the IOC by finding substances that have not been banned, using products that mask banned substances, or substances that cannot be tested for (Williams, 1995b). Currently, it is believed that the athletes who try to get away with the use of banned substances by far outnumber those who do not (Lamarine, 1998).

In recent years, the focus of ergogenic aid research has switched from pharmacological agents to nutritional factors (Williams, 1995b). Many athletes manipulate their diets, take supplements, or take substances that are normally found in the diet to try to improve their performance. Despite the widespread use of supplements, much of the research on their effectiveness reports conflicting results (Burke \& Heeley, 1994). One of these substances is caffeine, which is defined as a pharmacological agent, but is also an ingredient in most individuals' diets. It is easily available, inexpensive, and socially accepted. Caffeine is one of the oldest drugs currently used in modern society, and is found in many foods and beverages, but it has absolutely no nutritional value and is not necessary for any physiological processes (Clarkson, 1993).

Although the reported use of caffeine as an ergogenic aid dates back to the 1920's (Lamarine, 1998), it is only in the last two decades that caffeine has been studied as a potential ergogenic aid for sports performance. The majority of these studies concentrated on endurance exercise, due to one study that demonstrated a $20 \%$ improvement in time to exhaustion (Costill, Dalsky, \& Fink, 1978), and the general results have consistently indicated that caffeine improves performance (Tarnopolsky, 1994). However, the underlying mechanism behind these improvements in performance 
is not known and remains a continued topic of research and debate.

Recent investigations have begun to look at the effects of caffeine on high intensity exercise but results have been conflicting (Lamarine, 1998). In some, caffeine has been shown to increase time to exhaustion (Doherty, 1998; Jackman, Wendling, Friars, \& Graham, 1996; Trice \& Haymes, 1995) and improve race time during sprints and high intensity exercise up to 20 minutes in duration (Collomp, Ahmaïdi, Chatard, Audran, \& Préfaut, 1992; MacIntosh \& Wright, 1995; Wiles, Bird, Hopkins, \& Riley, 1992). Comparison of these studies is difficult due to the variability in factors such as individual responses to caffeine, habitual caffeine consumption, training status of participants, the type and mode of exercise, and diet (Burke \& Heeley, 1994).

The IOC has placed caffeine on its restricted substances list where a limit of $12 \mu \mathrm{g}$ of caffeine per ml urine is the criteria for banning athletes from competition (Clarkson, 1993). Levels of urine caffeine below this limit are permitted without any disciplinary action. Consumption of approximately five to six cups of strong coffee would cause an athlete to reach this threshold of $12 \mu \mathrm{g} / \mathrm{ml}$ (Williams, 1995a). However, studies of endurance exercise suggest that the ergogenic effects of caffeine tend to begin at much lower doses than those which are banned and that no additional effects are evident with larger doses (Clarkson, 1993; Graham \& Spriet, 1995). This indicates that caffeine has the potential of being a legal ergogenic aid under the IOC restrictions.

In a survey conducted by the Canadian Center for Drug Free Sport on 16, 000 Canadian athletes between the ages of 11 and 18 , it was found that $26.7 \%$ reported having used caffeine to enhance athletic performance within the last year (Graham, Rush, \& van Soeren, 1994). Due to this large number of athletes using caffeine to enhance 
performance, it is essential that research be undertaken to determine which types of exercise can benefit most from its ergogenic effects. Since so many sports consist of high intensity intermittent exercise (e.g. basketball, volleyball, football, hockey, soccer, rugby, etc.), it is important to determine whether ergogenic aids such as caffeine benefit performance during this type of exercise.

\subsection{Review of Literature}

\subsubsection{Caffeine}

Caffeine is a methyl xanthine derivative (1,3,7-trimethylxanthine) that is found in coffee, tea, cola drinks, chocolate, and analgesic preparations (Haynes \& Fitch, 1992). It occurs naturally in over 60 species of plants, including coffee beans, tea leaves, cocoa beans, cola nuts, and Guarana herb, and it is added to carbonated beverages and pharmaceuticals (Bucci, 1994). At room temperature caffeine is a white, odorless powder with a slightly bitter taste (Tarka \& Hurst, 1998).

Caffeine is the most commonly used drug in North America (Tarnopolsky, 1994) and coffee accounts for the majority of caffeine consumed (Lundsberg, 1998). In Canada, from 1990 to 1994, the average yearly consumption of coffee, tea, and cocoa per person was $4.4 \mathrm{~kg}, 0.5 \mathrm{~kg}$, and $2.1 \mathrm{~kg}$ respectively (Lundsberg, 1998). The average Canadian adult consumes $4 \mathrm{mg}$ caffeine per kg body weight per day (Smith \& Tola, 1998).

Once caffeine is ingested it is absorbed rapidly by the body and peak concentrations appear in the blood approximately 1 hour following ingestion (McArdle, 
Katch, \& Katch, 1991; Nehlig, Daval, \& Debry, 1992). Nearly 100\% of ingested caffeine is absorbed, although there is individual variation in the rate of gastric emptying and intestinal absorption (Tarnopolsky, 1994). The $\mathrm{pH}$ of the stomach affects the rate of absorption as well, where a higher $\mathrm{pH}$ increases the rate of absorption (Spiller, 1998). The half-life of plasma caffeine ranges from 3 to 10 hours and is longer in habitual caffeine consumers (Tarnopolsky, 1994). There are many metabolites of caffeine that circulate in the plasma that also have physiological effects (Spiller, 1998). The methylxanthines paraxanthine, theophylline, and theobromine all increase plasma free fatty acid levels and theophylline specifically affects cerebral circulation, increases electrical conduction in the heart, and stimulates the secretion of pancreatic hormones, which control organic metabolism and plasma glucose levels (Spiller, 1998). Once caffeine is absorbed, it is located primarily in body water with the highest concentrations found in skeletal muscle (Williams, 1991). The activity of the enzymes involved in the metabolism of caffeine can be influenced by diet, exercise, exposure to chemicals, disease, and age (Graham et al., 1994).

Caffeine is legally classified as a drug and has many powerful physiological effects on the human body (Nehlig et al., 1992; Spiller, 1998; Williams, 1995a). Side effects of caffeine include tremors, palpitations, restlessness, anxiety, headaches, irritability, diuresis, and gastro-intestinal symptoms (Haynes \& Fitch, 1992). Some gastro-intestinal effects include acid indigestion, heartburn, abdominal pain, gas, and constipation (Spiller, 1998). There is a large variability between individuals in the potential for adverse effects, which can only be specifically identified through trial and error. The diuretic effect causes some concern when caffeine is used during exercise due 
to the possibility of dehydration or heatstroke (Tarnopolsky, 1994). These can be caused by an increase in renal blood flow and glomerular filtration rate stimulated by caffeine, and an enhanced renin release from the kidneys (Spiller, 1998). It is generally assumed that moderate caffeine consumption or acute caffeine consumption does not cause any major health risks (Williams, 1995a). When less than 5 cups of coffee per day are consumed, there has been shown to be no increased risk of cancer, cardiovascular disease, or peptic ulcer disease (Tarnopolsky, 1994).

Caffeine increases metabolic heat production that, consequently, raises body temperature and increases the resting metabolic rate by up to $10 \%$ to $12 \%$ (Williams, 1995a). It can enter any system in the body and it is soluble in lipids so it can rapidly cross the blood-brain barrier by diffusion (Nehlig et al., 1992). Caffeine modifies the contractility of the heart and blood vessels, increases heart rate, and increases the nerve conduction velocity of the heart (Spiller, 1998). It influences central and peripheral nervous system neurotransmission by stimulating the central nervous system (Spiller, 1998). In the endocrine system, caffeine stimulates serotonin secretion in the cerebral cortex and cerebellum and causes a rise in plasma epinephrine and norepinephrine, secreted from the adrenal medulla (Spiller, 1998). Caffeine is also responsible for stimulating gastric secretion, gall bladder contraction, and pancreatic hormone secretions (Spiller, 1998).

\subsubsection{Potential Mechanisms for the Ergogenic Effect of Caffeine}

The stimulatory effect of caffeine may be caused by increasing muscle cell permeability to calcium, increasing cyclic adenosine monophosphate (cAMP) activity, or 
by blocking inhibitory adenosine receptors in the central nervous system, all of which would improve contractility (Haynes \& Fitch, 1992). Caffeine mobilizes intracellular calcium, lowering the excitability threshold of the muscle fiber and prolonging muscular contraction (Nehlig et al., 1992). It potentiates the release of calcium by the sarcoplasmic reticulum and inhibits its reuptake by the sarcoplasmic reticulum (Lamarine, 1998). In vitro, an increased tension of twitch contractions is well established (Lamarine, 1998; Nehlig et al., 1992; Tarnopolsky, 1994). An increased availability of calcium within skeletal muscle could potentially enhance muscle work. However, this mechanism does not seem plausible in human subjects since the caffeine dose required is $250 \mu \mathrm{M}$ of caffeine, which is between the toxic $(200 \mu \mathrm{M})$ and lethal $(500 \mu \mathrm{M})$ levels (Lamarine, 1998; Nehlig et al., 1992).

A second possible mechanism is an increased level of cellular cAMP caused by an inhibition of phosphodiesterases which generally functions to break down cAMP, inactivating the molecule (Nehlig et al., 1992). By inhibiting the activity of cyclic nucleotide phosphodiesterase, the enzymatic breakdown of cAMP is blocked (Lamarine. 1998). The release of cAMP is triggered by the activation of adenylate cyclase by $G$ protein and acts as a secondary messenger that diffuses throughout the cell having various effects by activating cAMP-dependent protein kinase which phosphorylates other proteins (Vander, Sherman, \& Luciano, 1990). These proteins are enzymes that become activated or inactivated when phosphorylated, changing their activity and causing various responses within the cell (Vander et al., 1990). The increased cAMP levels leads to increased lipolysis and therefore increased plasma free fatty acid levels (Lamarine, 1998). The greater free fatty acid availability has the potential to cause increased lipid oxidation 
and glycogen sparing (Tarnopolsky, 1994). If a greater amount of fat is used as fuel for exercise, it would take longer for glycogen depletion and subsequent fatigue to set in. Once again, however, the dose needed to cause this response is toxic to the human body (Lamarine, 1998). This does not mean that glycogen sparing is not a possibility, since caffeine increases epinephrine concentrations, which also increases lipid oxidation (Tarnopolsky, 1994).

Currently, the most scientifically accepted mechanism for caffeine's ergogenic effect is its competitive antagonism of adenosine receptors (Tarnopolsky, 1994). This occurs at physiological doses of caffeine that are less than $100 \mu \mathrm{M}$ and are non-toxic and have no effect on cAMP metabolism or calcium release (Nehlig et al., 1992). Adenosine has depressant effects on the central nervous system, such as the inhibition of neuronal electrical activity and the release of neurotransmitters (Lamarine, 1998). Caffeine, which is structurally similar to adenosine, inhibits the depressant effects of adenosine, causing a stimulant effect on the central nervous system, increased lipolysis in adipocytes, and increased gastric secretion (Tarnopolsky, 1994). When an antagonist binds to an adenosine receptor, the G protein is not activated, and cAMP is not released into the cell (Graham et al., 1994). Adenosine controls cardiac and smooth muscle, neurotransmitter release, and adipose tissue function (Graham et al., 1994). The increased lipolysis in adipocytes triggered by adenosine antagonism could also lead to glycogen sparing. The availability of free fatty acids is increased, which could increase their use as fuel for exercise. During endurance exercise, where glycogen depletion is a cause of fatigue, the increased use of free fatty acids as fuel for exercise may improve performance by sparing the body's glycogen stores. During higher intensity exercise, the improvement in 
performance may be due to a delay in the lactic acid build up normally caused by the use of glycogen as fuel for exercise

Caffeine increases the release of epinephrine and norepinephrine from the adrenal cortex (Spiller, 1998), and although the exact mechanisms responsible for this increase are uncertain, higher levels of these hormones can have several effects. It is currently believed that caffeine increases the secretion of epinephrine rather than its clearance from the blood (Graham \& Spriet, 1991). Several studies have found that during endurance and high intensity intermittent exercise, norepinephrine levels did not increase despite increased epinephrine levels (Graham \& Spriet, 1991; Greer, McLean, \& Graham, 1998; Lindinger, Graham, \& Spriet, 1993; Spriet et al., 1992) suggesting that caffeine acts on the sympathetic control of the adrenal medulla (Graham \& Spriet, 1991). Although it has been suggested that the increased epinephrine levels stimulate lipolysis and could contribute to glycogen sparing, epinephrine also has other effects (Graham \& Spriet, 1991). Epinephrine acts on adipocytes, liver, and muscle and stimulates both lipolysis and glycogenolysis (Bangsbo, Jacobsen, Nordberg, Christensen, \& Graham, 1992; Graham and Spriet, 1991), so epinephrine may also have the effect of increasing anaerobic metabolism because of the stimulation of glycogenolysis (Greer et al., 1998).

It has been suggested that the increased plasma epinephrine levels during exercise following caffeine ingestion may stimulate the $\mathrm{Na}^{+}-\mathrm{K}^{+}$pump activity and attenuate the potassium elevation in the blood (Lindinger et al., 1993). Potassium leaves the cell during muscle contraction and the $\mathrm{Na}^{+}-\mathrm{K}^{+}$pump is responsible for bringing potassium back into the cell (Lindinger et al., 1993). The increased extracellular potassium levels contribute to fatigue by lowering the contraction force and rate (Lindinger et al., 1993). 
Lindinger et al. (1993) demonstrated a smaller rise in plasma potassium concentration during endurance exercise following the ingestion of 3 to $9 \mathrm{mg}$ caffeine / $\mathrm{kg}$ body weight. However, Greer et al. (1998) did not demonstrate any effect of caffeine on potassium levels during repeated high intensity exercise following the ingestion of $6 \mathrm{mg}$ caffeine / kg body weight.

Finally, caffeine has the potential to act directly on the central nervous system by stimulating $\beta$-endorphin and serotonin release that can modify the perception of pain caused by physical exertion (Nehlig et al., 1992). This may enhance mood, improve the attitude toward exercise, and motivate athletes to work harder (Lamarine, 1998). However, there are no studies able to conclude whether caffeine can alter the perception of fatigue (Tarnopolsky, 1994).

\subsubsection{Caffeine and Endurance Exercise}

The majority of the research on caffeine as an ergogenic aid has looked at endurance exercise. Caffeine has been shown to increase the time to exhaustion (Cadarette, Levine, Berube, Posner, \& Evans, 1983; Costill et al., 1978; Graham \& Spriet, 1991; Sasaki, Maeda, Usui, \& Ishiko, 1987), decrease race time (Berglund \& Hemmingsson, 1982), and increase power output (Ivy, Costill, Fink, \& Lower, 1979). These are just a small sample of the studies on caffeine and endurance exercise. These studies have used athletic participants, tested performance variables such as time to exhaustion and race time, used a randomized study design, taken habitual caffeine consumption into account, and had participants abstain from caffeine prior to the protocol (Tarnopolsky, 1994). 
Costill et al. (1978) found a significant $19.5 \%$ increase in time to exhaustion during cycle ergometry at $80 \%$ of maximal oxygen consumption $\left(\mathrm{VO}_{2 \max }\right)$ in seven male and two female participants. Cadarette et al. (1983), who used four male and four female subjects, and Sasaki et al. (1987), who studied five male teenage athletes, found significant $37.4 \%$ and $33.0 \%$ increases in time to exhaustion respectively when participants ran on a treadmill at $80 \%$ of $\mathrm{VO}_{2 \max }$. Graham and Spriet (1991) used both cycle ergometry and treadmill running in seven elite athletes and found significant increases in time to exhaustion of $51 \%$ and $44 \%$ respectively. In addition, Berglund and Hemmingsson (1982) observed significant $1.7 \%$ and $3.2 \%$ decreases in race time in elite male and female skiers during Nordic ski races of approximately 21 kilometers. Finally, Ivy et al. (1979) found a significant $7.4 \%$ increase in power output in seven male and two female trained cyclists during a cycle ergometry test of two hours at $80 \mathrm{rpm}$ and with variable resistance.

\subsubsection{Caffeine and High Intensity Continuous Exercise.}

High intensity exercise is defined as "a form of continuous training performed at work intensities representing $85 \%$ to $95 \%$ of an athlete's maximum heart rate", near maximal oxygen uptake values (Wilmore \& Costill, 1994, p. 537). Studies on the effect of caffeine on high intensity exercise have used exercise protocols that range in time from 15 seconds (Williams, Signorile, Barnes, \& Henrich, 1988) to 25 minutes (MacIntosh \& Wright, 1995), and measured a variety of performance variables, blood factors, respiratory factors, and RPE. 
Berry, Stoneman, Weyrich, and Burney (1991) investigated the timing of the ventilatory and lactate thresholds during an incremental treadmill test in 10 male subjects. In this single-blind study, the treadmill grade was increased by $3 \%$ every minute, except for every third minute when the speed was increased. The participants ran until they reached their $\mathrm{VO}_{2 \max }$. They found that during the placebo trial the ventilatory and lactate thresholds occurred at the same time, however, during the caffeine trial the ventilatory threshold occurred at a significantly higher oxygen consumption $(44.4 \pm 2.1 \mathrm{~mL} / \mathrm{kg} / \mathrm{min})$ than the lactate threshold $(39.7 \pm 1.8 \mathrm{~mL} / \mathrm{kg} / \mathrm{min}$, Berry et al., 1991). During the caffeine trial the lactate threshold occurred at the same percentage of $\mathrm{VO}_{2 \max }$ as during the placebo trial, whereas the ventilatory threshold was higher in the caffeine trial. These results suggest that individuals may be able to reach their ventilatory threshold at a higher percentage of their $\mathrm{VO}_{2 \max }$ with caffeine. Since catecholamines affect ventilation during exercise, it is possible that the change in the ventilatory threshold is related to changes in epinephrine concentrations (Berry et al., 1991).

Three studies have focused on self-paced tasks of a specified distance (Collomp et al., 1992; MacIntosh \& Wright, 1995; Wiles et al., 1992). In a double-blind study, MacIntosh and Wright (1995) found that 7 male and 4 female participants significantly improved their time during a $1500 \mathrm{~m}$ swim following caffeine consumption. The total time improved to $20: 58.8 \pm 36.4$ minutes from $21: 21.8 \pm 38.2$ minutes, and the time of each $500 \mathrm{~m}$ interval also decreased. The subjects in this study chose to work at a higher intensity during the caffeine trial. Collomp et al. (1992) investigated 7 trained and 7 untrained swimmers, both male and female, on a $2 \times 100 \mathrm{~m}$ freestyle swim trial with 20 minutes of passive rest between the two sprints. In this double-blind protocol, they found 
that swimming velocity was significantly higher in the trained subjects during the caffeine trial, but there were no differences in performance in the untrained subjects (Collomp et al., 1992). The authors suggested that there are specific adaptations that occur during training that seem to be necessary for an ergogenic effect of caffeine to take place. One possible adaptation could be an enhanced lactic acid buffering capacity following anaerobic training (Collomp et al., 1992), however further research is needed. Lactic acid buffering would delay the build up of lactic acid in the muscles and therefore delay the onset of fatigue. Finally, Wiles et al. (1992) used a double-blind protocol for a $1500 \mathrm{~m}$ treadmill run in 18 trained male athletes. The subjects in this study ran significantly faster during the caffeine trial (286.0 seconds) than the placebo trial (290.2 seconds). In an additional trial, 10 trained male participants ran at a predetermined speed for $1100 \mathrm{~m}$ and picked a speed for the final minute of the run. They chose a faster speed during the caffeine trial $(23.5 \mathrm{~km} / \mathrm{h}$ compared to $22.9 \mathrm{~km} / \mathrm{h}$ ) for this finishing burst (Wiles et al., 1992). In these three studies the subjects may have had a decreased sensation of fatigue during the caffeine trial as compared to the placebo trial that prompted them to work at a higher intensity. In addition, if lipids provided a larger amount of fuel for exercise during the caffeine trial, the subjects would have been able to work harder before the build up of lactic acid from glycogen breakdown became a limiting factor.

Only one study to date has looked at the time to exhaustion during high intensity continuous exercise (Doherty, 1998). In a double-blind protocol, nine active male athletes had a significantly longer run time to exhaustion during the caffeine trial (208.2 \pm 13.6 seconds versus $181.0 \pm 9.4$ seconds) while running at $125 \%$ of their $\mathrm{VO}_{2 \max }$. At $125 \%$ of $\mathrm{VO}_{2 \max }$ the participants are using their sources of energy maximally (Doherty, 
1998). These subjects chose to run longer with the caffeine, despite the fact that they were working at exactly the same workload as during the placebo trial. The potential mechanisms that applied to the exercise of a set distance (pp. 12-13) also apply here.

The last two studies used protocols that had a finite time interval for the exercise period (Collomp, Ahmaïdi, Audran, Chanal, \& Préfaut, 1991; Williams et al., 1988). Collomp et al. (1991) had 3 male and 3 female untrained subjects perform the Wingate test, which is an all out cycle ergometer test for 30 seconds, against a load determined by body weight in a single-blind protocol. Caffeine did not effect anaerobic power, anaerobic capacity, and power decrease (Collomp et al., 1991). The authors believed increased epinephrine and norepinephrine levels indicated that the subjects had additional energy available to them during the caffeine trial, but were unable to convert it to mechanical energy since they were untrained individuals (Collomp et al., 1991). Untrained participants are unable to buffer lactic acid efficiently and reach fatigue independent of the availability of energy. In the Williams et al. (1988) study, 9 male subjects performed a maximal cycle for 15 seconds following a double-blind administration of caffeine or placebo. Williams et al. (1988) also found no significant differences in any of the performance variables of peak power, power fatigue index, total work, time to peak power, or power fatigue rate following caffeine ingestion. These studies suggest that caffeine has little effect on power output during high intensity exercise tasks.

The results from these studies on the effect of caffeine on high intensity continuous exercise suggest that caffeine improves performance only on specific types of tasks. During single exercise bouts, caffeine improved times on tasks with a set distance 
(Collomp et al., 1992; MacIntosh \& Wright, 1995; Wiles et al., 1992) and during exercise to exhaustion (Doherty, 1998), with no set distance or time. The tasks where caffeine does not seem to have an effect are those over a specified length of time (Collomp et al., 1991; Williams et al., 1988).

\subsubsection{Caffeine and High Intensity Intermittent Exercise}

High intensity intermittent exercise is defined as repeated bouts of exercise at a near maximal heart rate interspersed with rest periods. Both the lengths of the exercise periods and the rest periods can be varied to use different energy systems and thereby maximize performance results or obtain different training results. Studies on the effect of caffeine on high intensity intermittent exercise have used exercise protocols that range in time from 6 seconds (Anselme, Collomp, Mercier, Ahmaïdi, \& Préfaut, 1992) to 2 minutes (Jackman et al., 1996) and rest periods that range from 1 minute (Trice \& Haymes, 1995) to 6 minutes (Jackman et al., 1996). All of the studies involving high intensity intermittent exercise included both caffeine and placebo trials.

Caffeine ingestion prior to high intensity intermittent exercise has been investigated with respect to either time to exhaustion (Jackman et al., 1996; Trice \& Haymes, 1995) or to maximal anaerobic power (Anselme et al., 1992; Greer et al., 1998). Trice and Haymes (1995) alternated 1 minute of cycling at $85-90 \%$ of the maximum workload with one minute of rest for 30 minutes in a double-blind study with 8 trained male subjects. This cycle was repeated for up to three 30 minute sets with 5 minutes of rest in between each set or until the participant was unable to maintain the required 70 rpm for 15 seconds. Using this protocol, the time to exhaustion was significantly 
increased by 16.25 minutes during the caffeine trial when compared to the placebo trial. Jackman et al. (1996) used a slightly different protocol where the 3 female and 11 male recreational and varsity endurance athletes performed two sets of 2 minutes of cycling at $\mathrm{VO}_{2 \max }$ separated by 6 minutes of rest, followed by a third and final cycling bout until voluntary exhaustion. The caffeine and placebo administration was double-blind in this study. Time to exhaustion during the third cycling bout increased significantly from 4.12 \pm 0.36 minutes during the placebo trial to $4.93 \pm 0.60$ minutes during the caffeine trial. These results are similar to those obtained during single bouts of high intensity exercise for which it was also found that caffeine improved time to exhaustion (Doherty, 1998). A delay in the lactic acid build up and a decreased sensation of fatigue likely played a role in these studies as well.

The other studies that looked at high intensity intermittent exercise measured anaerobic power (Anselme et al., 1992; Greer et al., 1998). Ten male and four female participants performed 6 second maximum sprints against an increasing workload, with 5 minutes rest between each sprint in a double-blind protocol. This was continued until the subjects reached their maximal anaerobic power which was defined as the point at which an increase in workload did not result in any increase in anaerobic power (Anselme et al., 1992). They found that caffeine significantly increased maximal anaerobic power (964 Watts during the caffeine trial compared with 903.7 Watts during the placebo trial), but that the workload at which it was obtained did not differ between trials. It was shown that the caffeine resulted in an increased pedaling frequency at the final load (Anselme et al., 1992). Greer et al. (1998) used nine physically active subjects in a randomized double-blind protocol. Each subject performed four 30 second Wingate (maximal sprints 
on a cycle ergometer) trials each separated by four minutes of rest following either placebo or caffeine ingestion (Greer et al., 1998). There were no significant differences between the placebo and caffeine trials in peak power, average power, rate of power loss, or total power output (Greer et al., 1998). One of the possible reasons for no improvements in performance was that the participants were recreational athletes and therefore not anaerobically trained, making complete motor unit activation unlikely (Greer et al., 1998).

The results from these studies investigating the effect of caffeine on high intensity intermittent exercise suggest that caffeine has the potential to improve performance. Caffeine improved times during exercise to exhaustion (Jackman et al., 1996; Trice \& Haymes, 1995) with no set distance or time and increased pedaling frequency (Anselme et al., 1992) during exercise with a set time period. It still appears as though caffeine does not effect total power output of tasks with a pre-determined time period (Anselme et al., 1992; Greer et al., 1998).

\subsubsection{Caffeine and Perceived Exertion during Exercise}

The rating of perceived exertion (RPE) is a subjective rating that participants give to rate how hard they feel they are working (Wilmore \& Costill, 1994). The RPE is an integration of all the information that the individual is receiving from the musculoskeletal, cardiovascular, and respiratory systems (Borg, 1982). It is believed to correspond to other measures of intensity, such as heart rate and percentage of maximal oxygen consumption (Borg, 1982). Caffeine's stimulant effect on the central nervous 
system may alter the perception of fatigue, causing a decreased RPE at identical exercise intensities (MacIntosh \& Wright, 1995; Wiles et al., 1992).

Although caffeine has a generalized effect on the central nervous system, very few studies have measured RPE. MacIntosh and Wright (1995) and Wiles et al. (1992) both found no significant changes in RPE. This is important since both studies found significant improvements in performance. Participants decreased their times during a $1500 \mathrm{~m}$ swim (MacIntosh \& Wright, 1995) and a $1500 \mathrm{~m}$ treadmill run (Wiles et al., 1992). This suggests that these participants chose to swim or run at a higher intensity in order to finish the race faster during the caffeine trial, but they perceived themselves to be working at the same intensity as during the placebo trial (MacIntosh \& Wright, 1995;

Wiles et al., 1992).

The only study to look at the effect of caffeine on RPE during high intensity intermittent exercise did not report any significant effects (Trice \& Haymes, 1995). This is similar to the results from continuous exercise (MacIntosh \& Wright, 1995; Wiles et al., 1992) because this study also found an increased time to exhaustion during the caffeine trial when compared to the placebo trial (Trice \& Haymes, 1995). The participants chose to cycle for longer during the caffeine trial, but perceived themselves to be working the same length of time as without caffeine.

\subsubsection{Caffeine and Blood Lactate during Exercise}

Blood lactate is the most commonly investigated metabolite during caffeine studies. An increase in blood lactate during exercise indicates that anaerobic glycolysis is a major source of energy (Wilmore \& Costill, 1994). Glucose is broken down rapidly as 
a source of energy and has an end product of lactic acid that is converted to lactate and hydrogen ions (Wilmore \& Costill, 1994). However, blood lactate levels reflect levels of muscle glycogenolysis, muscle lactate release (Collomp et al., 1992), and the rate of lactate removal by other tissues, such as inactive muscle (Kowalchuk et al., 1988). This should be kept in mind when interpreting blood lactate data. Inactive skeletal muscle removes lactate from the blood and oxidizes it, and the lungs eliminate the carbon dioxide (Kowalchuk et al., 1988).

The majority of the studies on caffeine and high intensity exercise have not found any significant differences in maximum blood lactate levels (Berry et al., 1991; Doherty, 1998; MacIntosh \& Wright, 1995; Wiles et al., 1992). Two studies have found increases in maximal blood lactate taken via a finger prick (Collomp et al., 1992) and a catheter inserted in the cubital vein (Collomp et al., 1991). Collomp et al. (1991) observed significantly different blood lactate values of $8.8 \pm 0.96 \mathrm{mmol} / \mathrm{L}$ during the placebo trial compared to $11.47 \pm 0.88 \mathrm{mmol} / \mathrm{L}$ during the caffeine trial. Collomp et al. (1992) found significantly different blood lactate values of $9.3 \pm 0.8 \mathrm{mmol} / \mathrm{L}$ in the placebo trial and $10.9 \pm 0.8 \mathrm{mmol} / \mathrm{L}$ in the caffeine trial in the untrained subjects and $13.1 \pm 0.2 \mathrm{mmol} / \mathrm{L}$ in the placebo trial and $15.5 \pm 0.5 \mathrm{mmol} / \mathrm{L}$ in the caffeine trial in the trained subjects. It is possible that the studies that found no differences in blood lactate used exercise tasks that lasted long enough to involve increased lipolysis and potential glycogen sparing. Therefore, an increase in blood lactate would not be expected. The studies that found changes in blood lactate during the caffeine trial were of relatively short duration (30 second Wingate test, Collomp et al. 1991; 2 x 100 m swim, Collomp et al., 1992). Since caffeine stimulates the release of epinephrine, which increases glycolysis, blood lactate 
would increase when the duration of exercise is short enough to use a predominantly anaerobic energy supply.

The effect of caffeine on blood lactate during high intensity intermittent exercise seems to be similar to that found during single bouts of high intensity exercise. Three of the studies found no significant differences in blood lactate levels between the caffeine and placebo trials (Greer et al., 1998; Jackman et al., 1996; Trice \& Haymes, 1995). However, Anselme et al. (1992), with repeated 6 second sprints, observed a significant increase in blood lactate at the end of the exercise session $(8.36 \pm 0.95 \mathrm{mmol} / \mathrm{L}$ during caffeine trial and $7.17 \pm 0.53 \mathrm{mmol} / \mathrm{L}$ during placebo trial) as well as after 5 minutes of recovery $(10.23 \pm 0.97 \mathrm{mmol} / \mathrm{L}$ during caffeine trial and $8.35 \pm 0.66 \mathrm{mmol} / \mathrm{L}$ during placebo trial). This is similar to the two single bout studies that measured anaerobic power and also reported increases in blood lactate during the caffeine trial (Collomp et al., 1991; Collomp et al., 1992).

All of the studies that measured time to exhaustion (Doherty, 1998; Jackman et al., 1996; Trice \& Haymes, 1995) or time over a set distance (MacIntosh \& Wright, 1995; Wiles et al., 1992) found no differences in blood lactate between the caffeine and placebo trials despite the achievement of higher exercise intensities during the caffeine trials. This suggests that increased lipolysis and subsequent delay in lactic acid build up are potential mechanisms for the ergogenic effect of caffeine during high intensity exercise.

\subsubsection{Caffeine and Respiratory Factors during Exercise}

A few studies have investigated respiratory factors such as $\mathrm{VO}_{2}$, carbon dioxide production $\left(\mathrm{VCO}_{2}\right)$, and the respiratory exchange ratio (RER) during exercise (Berry et 
al., 1991; Doherty, 1998; Wiles et al., 1992), but, generally, the results have been inconclusive. The RER is the ratio of oxygen consumed to carbon dioxide produced $\left(\mathrm{VCO}_{2} / \mathrm{VO}_{2}\right)$. Since lactate accumulation increases carbon dioxide production (Wilmore \& Costill, 1994), the RER is generally higher during high intensity exercise that involves anaerobic glycolysis. If an increase in lipolysis occurs following caffeine consumption, then it would be expected that the RER would be lower during the caffeine trial when compared to the placebo trial. A RER of 0.7 indicates that fat is the major source of energy, while a RER of 1.0 indicates that carbohydrates are the major sources of energy (Williams, 1995a). During intense exercise, RER often goes over 1.0, indicating that the amount of carbon dioxide being produced is greater than the amount of oxygen being consumed. When this occurs, the body is using primarily anaerobic metabolism (Vander et al., 1990). If glycogen sparing were a factor, it would be expected that the RER would be lower during the caffeine trial. Two studies have measured RER and one reported no significant change between the caffeine and placebo trials (Berry et al., 1991), while the other reported a significantly lower RER during the caffeine trial (Wiles et al., 1992). These conflicting results make it difficult to draw any conclusions with respect to RER.

Some of the other respiratory variables that can be measured during exercise include $\mathrm{VO}_{2 \max }, \mathrm{VCO}_{2}$, ventilation volume (VE), and the maximal accumulated oxygen deficit. Berry et al. (1991) found no significant differences between the caffeine and placebo trials for any of the respiratory variables that they measured $\left(\mathrm{VO}_{2 \max }, \mathrm{VCO}_{2}, \mathrm{VE}\right)$, while Doherty (1998) observed a significantly greater maximal accumulated oxygen deficit during the caffeine trial during running at $125 \%$ of $\mathrm{VO}_{2 \max }$. Anaerobic glycolysis is the major source of energy used to make-up the oxygen debt during high intensity 
exercise, indicating an increase in the use of this energy system during the caffeine trial (Doherty, 1998). Once again, it is difficult to draw conclusions from the contradictory results of these studies.

None of the studies that have investigated high intensity intermittent exercise measured any respiratory factors (Anselme et al., 1992; Trice \& Haymes, 1995; Jackman et al., 1996). From the results of those studies that have looked at respiratory factors, it is difficult to make any conclusions regarding the effect of caffeine during exercise. Further measurement of respiratory factors, such as RER, is needed.

\subsubsection{Caffeine Dose}

There are two studies investigating the effect of varying doses of caffeine on endurance exercise (Graham \& Spriet, 1995; Pasman, van Baak, Jeukendrup, \& de Haan, 1995). Graham \& Spriet (1995) used eight male distance runners to compare 3, 6, or 9 $\mathrm{mg}$ caffeine $/ \mathrm{kg}$ body weight during a run to exhaustion at $85 \%$ of their $\mathrm{VO}_{2 \max }$. They found a progressive increase in the amount of caffeine metabolites in the plasma between the 3 and $6 \mathrm{mg} / \mathrm{kg}$ trials. However, there was no additional increase following the 9 $\mathrm{mg} / \mathrm{kg}$ dose, suggesting that the enzymes involved in caffeine metabolism may become saturated (Graham \& Spriet, 1995). Caffeine significantly increased time to exhaustion during the caffeine trials with the 3 and $6 \mathrm{mg} / \mathrm{kg}$ doses $(22.0 \pm 9.0 \%$ and $21.9 \pm 7.2 \%$ respectively). There were no significant differences in time to exhaustion between the 3 and $6 \mathrm{mg} / \mathrm{kg}$ doses. However, the $9 \mathrm{mg} / \mathrm{kg}$ dose showed no significant improvement in performance $(10.9 \pm 12.6 \%$, Graham \& Spriet, 1995). These results suggest that there are 
no additional ergogenic effects of caffeine on endurance exercise performance above caffeine doses of 3 to $6 \mathrm{mg} / \mathrm{kg}$.

Pasman et al. (1995) compared doses of 5, 9, and $13 \mathrm{mg}$ caffeine / $\mathrm{kg}$ body weight in nine well-trained subjects during cycling to exhaustion at $80 \%$ of maximal power output. These researchers found no significant differences in performance among the three caffeine doses, although they were all significantly different from the placebo trial (average of $27 \%$ increase in time to exhaustion, Pasman et al., 1995). This study also took urine samples following exercise and found urine caffeine concentrations of $4.8 \pm$ $1.8,8.9 \pm 5.2$, and $14.9 \pm 6.9 \mu \mathrm{g} / \mathrm{mL}$ for the 5,9 , and $13 \mathrm{mg} / \mathrm{kg}$ doses respectively (Pasman et al., 1995). Only the $13 \mathrm{mg} / \mathrm{kg}$ dose produced urine caffeine levels above the limit set by the IOC, even though all three doses provided similar increases in exercise performance.

The studies on high intensity exercise and caffeine have used caffeine doses of 5 $\mathrm{mg} / \mathrm{kg}$ (Collomp et al., 1991; Doherty, 1998; Trice \& Haymes, 1995), $6 \mathrm{mg} / \mathrm{kg}$ (Greer et al., 1998; Jackman et al., 1996; MacIntosh \& Wright, 1995), and $7 \mathrm{mg} / \mathrm{kg}$ (Berry et al., 1991; Williams et al., 1988). A few studies have used a set dosage of caffeine (Anselme et al., 1992; Collomp et al., 1992; Wiles et al., 1992), but doses assigned relative to body weight are more prevalent.

\subsubsection{The Effect of Habitual Caffeine Consumption}

Chronic caffeine use may lead to caffeine habituation and blunting of the usual metabolic responses (Bucci, 1993). Chronic caffeine consumption has been shown to increase the number of adenosine receptors (Nehlig et al., 1992) so caffeine would be less 
likely to competitively bind with all the receptors. If caffeine's actions involve antagonism of adenosine, an increase in adenosine receptors would result in a decreased effect of caffeine since more receptors would remain available to bind with adenosine (Nehlig et al., 1992). However, the central nervous system becomes less tolerant to the effects of caffeine than other systems, such as the gastro-intestinal system (Nehlig et al., 1992). Research is further complicated by a difficulty in distinguishing the development of tolerance from low sensitivity to caffeine (Nehlig et al., 1992). While certain individuals may demonstrate a blunting of the usual response to caffeine following chronic consumption, others may not have been sensitive to the effects of caffeine to begin with and therefore do not demonstrate a habituation effect. However, differences in habitual caffeine intake may play a part in the individual variations in responses that are generally seen.

None of the studies investigating the effect of caffeine on high intensity exercise have compared different levels of habitual caffeine consumption. Two studies using endurance exercise performance compared results between caffeine users and nonusers (Dodd, Brooks, Powers, \& Tulley, 1991; van Soeren, Sathasivam, Spriet, \& Graham, 1993). Dodd et al. (1991) used seventeen moderately trained men during a graded cycle ergometer test to exhaustion. These researchers defined caffeine users as those consuming more than $300 \mathrm{mg} /$ day and nonusers as consuming less than $25 \mathrm{mg}$ /day (Dodd et al., 1991). van Soeren et al. (1993) studied fourteen untrained males during one hour of cycle ergometer exercise at $50 \%$ of $\mathrm{VO}_{2 \max }$. Nonusers were defined as consuming $0-20$ mg caffeine / week and users as consuming 400-750 mg/day (van Soeren et al., 1993). 
Neither of these studies found any significant differences in exercise performance between the caffeine users and nonusers.

Some studies have shown the epinephrine response to caffeine to differ between habitual caffeine users and non-users (Bangsbo et al., 1992; van Soeren et al., 1993). Bangsbo et al. (1992) found epinephrine and norepinephrine levels were significantly lower during endurance exercise following six weeks of chronic caffeine consumption than before. van Soeren et al. (1993) demonstrated no significant differences in epinephrine levels between the placebo and caffeine trials in caffeine users and higher levels during the caffeine trials in non-caffeine users. In this study, the caffeine users' epinephrine levels during both trials were similar to the levels of the non-users during their caffeine trial (van Soeren et al., 1993). However, another study has shown that habitual caffeine consumption had no effect on plasma epinephrine levels (Graham \& Spriet, 1991).

Most of the studies investigating the effect of caffeine on high intensity exercise that have assessed habitual caffeine consumption have used participants who were not habitual caffeine consumers (Berry et al., 1991; MacIntosh \& Wright, 1995; Trice \& Haymes, 1995; Williams et al., 1988). Doherty (1998) used a range of caffeine users (30-850 $\mathrm{mg} / \mathrm{day})$, and found no pattern between the changes in exercise performance and habitual caffeine consumption. 


\subsection{Statement of the Problem and Hypotheses}

\subsubsection{Statement of the Problem}

Very few studies have investigated the effects of caffeine ingestion on high intensity intermittent exercise performance (Anselme et al., 1992; Greer et al., 1998; Jackman et al., 1996; Trice \& Haymes, 1995). These studies have shown no consistent pattern, but tend to suggest that caffeine may enhance exercise performance during this type of exercise. Jackman et al. (1996) and Trice and Haymes (1995) both found increases in time to exhaustion during the caffeine trial while Anselme et al. (1992) and Greer et al. (1998) found no differences in power output. Differences with these studies have included variability in the training status of the subjects, habitual caffeine consumption, and diet.

The purpose of this study, therefore, was to determine the effect of acute caffeine consumption on the performance of high intensity intermittent exercise while controlling for training status, habitual caffeine consumption, and diet. The effect of caffeine on time to exhaustion and rating of perceived exertion was investigated during repeated sprints on a treadmill. In addition, the effect of caffeine on blood lactate and the respiratory exchange ratio was explored during both exercise and recovery. Habitual exercise levels, habitual caffeine consumption, and food intake prior to each test were assessed as potential confounding variables. 


\subsubsection{Hypotheses}

The major hypothesis was that:

1. There would be an increase in the time to exhaustion during high intensity intermittent exercise during the caffeine trial as compared to the placebo trial.

The secondary hypotheses were that:

1. The increase in time to exhaustion would be associated with a lower rating of perceived exertion during the caffeine trial as compared to the placebo trial.

2. Blood lactate levels would be higher following the caffeine trial as compared to the placebo trial due to a longer exercise period.

3. Blood lactate levels would be lower following the third sprint, due to an increase in free fatty acid oxidation as fuel for exercise during the caffeine trial as compared to the placebo trial.

4. The respiratory exchange ratio would be lower during the caffeine trial as compared to the placebo trial, reflecting a greater utilization of fats as fuel for exercise following caffeine ingestion.

The exploratory objective was that:

1. The ventilation volume and heart rate values would be investigated to determine if any differences exist between the caffeine and placebo trials.

\subsubsection{Limitations}

1. Results obtained from this study can only be applied to the specific population subjects were drawn from. 
2. The primary measure of performance was the time run to voluntary exhaustion, which depends on the motivation of the individual subjects. If a participant was differently motivated on subsequent testing occasions there may have been some impact on the results.

3. Dietary habits could not be completely controlled since the subjects were free-living persons. Food intake estimation relies upon the ability of the subjects to report accurate portion sizes, as well as their loyalty in filling out the three-day food record.

\subsubsection{Delimitations}

1. Results from this study apply to the specific age range of the subjects, their training status, and their caffeine intake since all of these variables may affect the results.

2. Since this study was conducted in a laboratory situation, it is difficult to predict whether caffeine would have similar effects in a field setting. 


\section{CHAPTER 2}

\section{METHODS}

\subsection{Research Design}

This study used a double-blind repeated measures design in which every subject was involved in both a caffeine and placebo treatment condition. All subjects were required to come to the laboratory four times, twice for familiarization and twice for data collection. The treatment was either placebo or caffeine and the dependent variables were (1) time to exhaustion, (2) rating of perceived exertion, (3) blood lactate, (4) respiratory exchange ratio, (5) ventilation volume, and (6) heart rate. In addition, normal physical activity levels, habitual caffeine consumption, and actual dietary intake prior to each testing occasion were assessed to control for these potential confounding variables.

\subsection{Participants}

Nineteen participants ( 9 male, 10 female) were used in this study. Both male and female participants were included since studies conducted to date have included only males (Berry et al., 1991; Doherty, 1998; Trice \& Haymes, 1995; Williams et al., 1988; Wiles et al., 1992) or mostly males (Jackman et al., 1996; MacIntosh \& Wright, 1995). The two studies that did use both males and females found no gender differences in the 
effect of caffeine on performance (Jackman et al., 1996; MacIntosh \& Wright, 1995). Nevertheless, it has been shown that, males and females do differ in some metabolic aspects of exercise (Tarnopolsky, MacDougall, Atkinson, Tarnopolsky, \& Sutton, 1990). Females tend to use more intramuscular triglycerides whereas males use more glycogen as fuel for exercise (Tarnopolsky et al., 1990). Thus, in this study, the data will first be analyzed comparing males and females to investigate any differences in substrate utilization.

Since evidence suggests that the ergogenic effects of caffeine during high intensity exercise may be limited to trained individuals, defined by training five to six days per week (Collomp et al., 1992), participants were recruited from competitive intercollegiate volleyball, basketball, and football teams at the University of Saskatchewan. These sports were chosen since they involve high intensity intermittent exercise. The descriptive characteristics of the seventeen participants whose data was analyzed (9 males, 8 females) are presented in Table 2.1 . 
Table 2.1

Participant descriptive characteristics (mean \pm standard deviation)

\begin{tabular}{lcc}
\hline Characteristic & Males $(n=9)$ & Females $(n=8)$ \\
\hline Age (years) & $22.0 \pm 2.9$ & $21.4 \pm 2.0$ \\
Height $(\mathrm{cm})$ & $184.8 \pm 7.2$ & $176.0 \pm 6.5^{*}$ \\
Weight $(\mathrm{kg})$ & $84.8 \pm 4.4$ & $69.8 \pm 5.3^{* *}$ \\
Daily caffeine intake (mg) & $149.8 \pm 121.2$ & $197.6 \pm 206.9$ \\
Weekly physical activity (METS) & $71.0 \pm 44.7$ & $56.4 \pm 39.3$ \\
\hline
\end{tabular}

*males $>$ females $(p<.05)$.

$* *$ males $>$ females $(p<.01)$.

\subsection{Procedures}

\subsubsection{Test Protocol}

The researcher recruited participants by attending team practices, explaining the requirements of the study, and circulating a sign up sheet, as well as recruiting by phone using a team list supplied by the coaches. Participants were informed as to the nature of the study and what would be required of them, and written consent was obtained (Appendix A). During the first visit to the laboratory, participants were given a three-day food record (Appendix B) to be completed for the three days prior to each testing day. In addition, they were instructed to abstain from caffeine containing beverages and food products for a minimum of 72 hours prior to the testing trial days (Appendix $\mathrm{C}$ ). They were also instructed to abstain from anaerobic activity for 48 hours prior to testing and 
from aerobic activity for 24 hours (Appendix C), which are the recommended time periods for full recovery from these types of exercise to occur (Stone \& Conley, 1994). Participants were instructed to eat as they normally would on the testing days up until two hours prior to the test, after which only water was permitted. Due to the strenuous, exhaustive nature of the protocol, a longer fasting period would not have been appropriate. Compliance to these conditions was confirmed using a checklist (Appendix D) once the participant arrived at the laboratory.

Each participant was required to attend the laboratory on four separate occasions. At the first session, they learned how to run on the treadmill. The second session was a habituation procedure, and the third and fourth sessions were the testing trials during which the caffeine or placebo doses were given. All testing took place in the Exercise Physiology Laboratory in the College of Kinesiology at the University of Saskatchewan. The University of Saskatchewan's Advisory Committee on Ethics in Human Experimentation approved this study. The Dean's Research Fund of the College of Kinesiology covered the costs of the caffeine and placebo capsules and the laboratory supplies required for the study. No financial incentives were provided to any of the participants. The College of Pharmacy and Nutrition at the University of Saskatchewan manufactured the caffeine and placebo capsules. Caffeine capsules were manufactured using pharmaceutical grade caffeine (Wiler Fine Chemicals Ltd., London, Ont.) and the placebo contained an equivalent amount of lactose in opaque capsules identical in size and appearance to the caffeine capsules. 


\subsubsection{Physical Activity Measurement}

The Leisure Time Exercise Questionnaire (Godin \& Shephard, 1985, Appendix E) was used to assess habitual physical activity levels in the participants. This questionnaire provides a score calculated in metabolic equivalents (METs) expended per week by the individual, which represents multiples of the resting metabolic rate (Williams, 1995a). METs can be converted to provide an estimate of energy expenditure in calories and oxygen consumption in $\mathrm{L} / \mathrm{min}$. A score is given for strenuous, moderate, and mild exercise depending on how often each of these types of exercise is undertaken each week. The times per week of strenuous exercise were multiplied by 9 METs, moderate exercise by 5 METs, and mild exercise by 3 METs (Godin \& Shephard, 1985).

The test-retest reliability of this questionnaire is 0.94 for strenuous exercise, 0.46 for moderate exercise, and 0.48 for light exercise (Godin \& Shephard, 1985). Concurrent validity testing demonstrated that $\mathrm{VO}_{2 \max }$ was significantly correlated $(\mathrm{r}=0.38)$ to strenuous exercise (Godin \& Shephard, 1985).

\subsubsection{Habitual Caffeine Consumption Measurement}

Habitual caffeine consumption was determined using the estimation of habitual caffeine consumption questionnaire (Trice \& Haymes, 1995, Appendix F). The questionnaire consists of a list of caffeine containing products from which the subjects indicate how often they consume each item; it was modified to contain only the products that are available in Canada. The amount of caffeine in each product is used to determine an average daily caffeine intake. While there is no standard questionnaire used to estimate habitual caffeine consumption, this type of questionnaire is similar to those other 
researchers have used to estimate habitual caffeine consumption (Engels \& Haymes, 1992; van Soeren et al., 1993; Wiles et al., 1992).

\subsubsection{Dietary Assessment}

Dietary intake was recorded for three days prior to each testing occasion to monitor the possible effects that a high or low carbohydrate diet can have on high intensity exercise performance. Participants used a three-day food record booklet (Appendix B) to record what they ate each day so that actual intake could be determined. They were instructed to carry the booklet with them and record what they ate and the amount of each product. The WEST dietary analysis program (Diet Analysis Plus Version 1.1, West Publishing Company, St. Louis, MI, 1994) was used to analyze the food records. Each item eaten was entered and the program provided the total number of calories consumed on average over the three days, as well as the amount of energy from carbohydrates, fats, proteins, and alcohol (Appendix G). This information was collected to determine whether diet might be a confounding factor in this study.

\subsubsection{Treadmill Accustomization}

The goal of the first laboratory visit was to make the participant familiar with running on the treadmill at the speed and incline to be used during testing. Prior to getting on the treadmill all participants completed the Physical Activity Readiness Questionnaire (PAR-Q, Appendix H) to ensure they would be able to safely participate in the testing protocol and read and signed the informed consent form (Appendix A). Participants also filled out the background information questionnaire (Appendix I), 
Leisure Time Exercise Questionnaire (Godin \& Shephard, 1985, Appendix E), and the Estimation of Habitual Caffeine Consumption Questionnaire (Trice \& Haymes, 1995, Appendix F). Participants were first taught how to get on and off of the treadmill and then underwent the sprinting protocol exactly as it would be performed during the testing trials, except they were not required to ingest either the placebo or caffeine capsules. In this first accustomization trial they did not wear the heart rate monitor, the facemask for respiratory measurements, nor have blood taken for lactate analysis.

\subsubsection{Habituation Procedure}

The second visit was a habituation procedure during which participants went through the testing procedures exactly as described for the testing trials except that participants were not required to ingest either the placebo or caffeine capsules. Participants began their warm-up immediately upon arrival at the laboratory and followed the procedures outlined for the testing trials described below.

\subsubsection{Testing Trials}

The final two visits were the testing trials during which caffeine or a placebo was administered in a randomized, double-blind order. Randomization was performed using the roll of a die where odd numbers meant that they received the placebo and even numbers the caffeine. Upon arrival at the laboratory, height and weight were recorded for each participant and one of the testers determined the dose of the drug the participant was to receive $(6 \mathrm{mg} / \mathrm{kg} *$ body weight in $\mathrm{kg})$. They were immediately given either the caffeine or placebo capsules by an individual who was not involved in any other part of 
the testing. None of the testers or participants had access to the double-blind key until all of the testing was competed. Participants waited for one hour before testing began to allow for the absorption of caffeine and its distribution throughout the body (Nehlig et al., 1992). During this time the preparation checklist on the data recording sheet (Appendix D) was reviewed with each participant to ensure that he or she had remembered to abstain from caffeine for the previous 72 hours, anaerobic exercise for 48 hours, aerobic exercise for 24 hours, and food for 2 hours. The subject's food record (Appendix B) for the previous three days was also collected.

During all of the testing trials three testers were present. One was responsible for running the metabolic cart (Sensor Medics Vmax29 Series, Anaheim, CA) and recording the data, the second for operating the treadmill and timing the sprints, and the third stood behind the participants while they were running to catch the participant in case they stumbled or tripped and also took the blood samples. All testers were given a copy of the study protocol (Appendix $\mathrm{J}$ ) to ensure that the protocol was followed precisely on every testing occasion.

Following the one hour waiting period, participants were fitted with a polar heart rate monitor (Polar Favor, Polar CIC Inc., Port Washington, NY). This is a belt containing electrodes that is strapped around the participant's chest. The electrodes pick up the electrical signal of the heart and transmit it to an external receiver that is connected to the computer, so that the heart rate data can be saved in the same file with the respiratory data. Heart rate data were recorded and saved as an average value over each 10 second time interval throughout each trial. The metabolic cart software (Sensor Medics Vmax29 Series, Anaheim, CA) set this averaging interval. 
Participants warmed up on the treadmill for 5 minutes at a speed of $6 \mathrm{mph}$ to elicit a heart rate around 150 bpm (Canadian Society for Exercise Physiology [CSEP], 1996), the standard warm-up for the Cunningham and Faulkner (1969) treadmill test. Following warm up, participants stretched for 5 minutes with care taken to stretch all of the major muscle groups in the legs. During the warm-up, the metabolic cart was calibrated so that gas collection could start as soon as the participant was ready. The metabolic cart was calibrated for both flow and gas concentrations following the procedures outlined in the operating manual (SensorMedics Corporation, 1995).

Following the 5 minutes of stretching, a facemask (Hans Rudolph Inc., 8940 Series, Kansas City, MO) for collection of expired air was put on the participant and hooked up to the metabolic cart so expired gases could be collected and analyzed. Respiratory and heart rate baseline data were collected for 5 minutes before exercise began. The participants stood still for the first 3 minutes of this baseline collection then walked for the final two minutes at $3 \mathrm{mph}$ so that they did not start sprinting from a stationary position. Expired gases were analyzed for $\mathrm{VCO}_{2}, \mathrm{VO}_{2}$, ventilation volume, and determination of the respiratory exchange ratio. The results were printed using 10 second averages set by the software in order to use uniform intervals (Appendix $\mathrm{K}$ ).

The exercise portion of the protocol involved a modification of the Cunningham and Faulkner treadmill test (Cunningham \& Faulkner, 1969), where the treadmill was set at a $20 \%$ grade and a speed of $7.5 \mathrm{mph}$. The Cunningham and Faulkner (1969) protocol allows the choice of a speed between 7 and $8 \mathrm{mph}$, and $7.5 \mathrm{mph}$ was chosen for this study. The original protocol involved choosing a different speed for each individual to elicit exhaustion within 30 to 60 seconds (Cunningham \& Faulkner, 1969). This protocol 
has a test-retest reliability that ranges from $r=0.76$ to 0.91 (Bouchard, Taylor, Simoneau, \& Dulac, 1991). In this study, the speed of $7.5 \mathrm{mph}$ was chosen to keep a uniform protocol for all participants while maintaining as many participants as possible within this time range. Pilot testing on four participants determined that this was a suitable speed. Participants performed three 30 second sprints at $7.5 \mathrm{mph}$. During each sprint, the tester counted every 5 second interval out loud so the participant would know how much time remained.

Immediately upon the completion of each sprint, the participant was asked for his or her RPE using the Borg scale (Borg, 1982, Appendix E). The participant was asked to look at the scale, which was posted on the wall in front of the treadmill, and give a rating as to how hard each individual felt he or she was working. The scale is a ratio scale that ranges from 6 to 20 where 7 is very, very light and 19 is very, very hard (Borg, 1982).

The sprints were separated by 2 minutes of walking on the treadmill at $3.5 \mathrm{mph}$ and a 5\% incline. The speed and incline of the treadmill for the recovery periods were chosen due to the length of time that it took to raise and lower the treadmill (about 15 seconds). Two minutes was chosen to allow participants to recover from the sprint enough to be able to complete the next sprint, but allow for the exercise to become progressively more difficult so they would be nearing exhaustion on the final sprint. During the raising and lowering of the treadmill participants straddled the treadmill belt. The first blood sample for lactate analysis was collected after the third 30 second sprint.

A fourth and final sprint consisted of a run to exhaustion with the time taken to exhaustion being recorded. Timing began when participants let go of the railing at the beginning of their sprint and stopped when they grabbed back onto the railing to get off 
the treadmill when they felt they could no longer continue. During this final sprint, the participants were not given any indication of the length of time that they had been running; they only received positive encouragement from all of the testers to continue for as long as possible. All three testers continually provided verbal encouragement for the entire time that the participant was running. Participants were also not told their time after the completion of the sprint. A second blood sample was taken immediately after this final sprint to exhaustion. Following this final sprint, participants walked on the treadmill at a $0 \%$ incline for a 5 minute recovery period. At the end of the 5 minute recovery period a third and final blood sample was taken, then all data recording ended, but participants continued to walk until they felt they had completely recovered.

Blood lactate was measured using a blood lactate analyzer (YSI 2300 Stat Llactate analyzer) using blood obtained from a finger prick on a finger of the participant's choice on his or her non-dominant hand. The finger was pricked using a hand held lancet and blood was collected in a heparinized capillary tube (Natelson blood collecting tube). The blood was immediately transferred into a microcontainer (Microtainer with lithium heparin) and whole blood was analyzed for blood lactate using the YSI 2300 Stat Llactate analyzer within a few minutes (Appendix M). This analyzer uses L-lactate oxidase within a membrane, which oxidizes the lactate in the blood, producing hydrogen peroxide. A platinum anode oxidizes the hydrogen peroxide, producing an electron flow proportional to the amount of blood lactate (Yellow Springs Instrument Company, 1991). The lactate membrane was checked prior to testing every day for accuracy using 3 $\mathrm{mmol} / \mathrm{L}$ and $10 \mathrm{mmol} / \mathrm{L}$ standards, which were prepared from laboratory grade chemicals. The membrane was changed when its accuracy moved beyond $\pm 0.3 \mathrm{mmol} / \mathrm{L}$. 
Most participants required 2-3 finger pricks to obtain the three blood samples, but an effort was made to use as few pricks as possible.

\subsubsection{Capsule Administration}

Once the investigator had determined the dose of the drug, the data sheet was given to a laboratory assistant not directly involved in any of the other testing. The dose given was approximately $6 \mathrm{mg}$ caffeine per kg body weight (Jackman et al., 1996; MacIntosh \& Wright, 1995). This dose has been shown to consistently produce urine caffeine levels below the International Olympic Committee threshold of $12 \mu \mathrm{g} / \mathrm{L}$ urine (Graham \& Spriet, 1995). Since the form of caffeine was $100 \mathrm{mg}$ capsules, the dose was rounded to the nearest $100 \mathrm{mg}$. The doses ranged from $400 \mathrm{mg}$ to $600 \mathrm{mg}$. The average dose for males was $511 \mathrm{mg}$ and $430 \mathrm{mg}$ for females. This is equivalent to 3-6 cups of coffee since one cup of coffee contains approximately 100-150 mg of caffeine (Williams, 1995a).

\subsection{Statistical Analyses}

Data collected were entered into a database. Averages were obtained for each variable for the baseline, sprints one through four, and recovery periods one through four. These values were then exported into the statistical analysis program (SPSS, version 7.5.1, SPSS Inc., Chicago, IL) for further analyses.

Baseline values for respiratory exchange ratio, ventilation volume, and heart rate were determined using a one minute average from minute two to minute three of the five minute baseline period, during which the subject was standing still. Sprint values, 
converted to $\mathrm{L} /$ minute (VE) and beats/minute (heart rate), for these variables were determined using an average for the 30 second period for sprints one through three. Sprint four values were also determined using 30 second averages from 0 to 30 seconds, 31 to 60 seconds, and 61 to 90 seconds, depending on the length of time that each subject ran. Recovery values following the first three sprints were determined by using values averaged over the final 30 seconds of the two minute recovery periods. Final recovery values were determined by averaging the last minute of the five minute recovery period.

A repeated measures analysis of variance (ANOVA), with treatment as the independent variable was used to determine whether there were any differences between the caffeine and placebo trials for the dependent variables. The data were analyzed as one group as well as by a two factor (gender $\mathrm{x}$ treatment) ANOVA. Participants' physical activity levels and habitual caffeine consumption were entered as covariates into a repeated measures analysis of covariance (ANCOVA) to determine whether these factors had any effect on the relationship. A $2 \times 3$ (treatment $x$ sprint) repeated measures ANOVA was used to determine whether there were any differences between the caffeine and placebo trials during the first three sprints for rating of perceived exertion, RER, VE, and heart rate. Friedman's non-parametric test was used to determine whether any of the identified trends were significant. Statistical significance was set at $p \leq 0.05$ for all tests. 


\section{CHAPTER 3}

\section{RESULTS AND DISCUSSION}

\subsection{Results}

The purpose of this study was to determine the effect of acute caffeine ingestion on the performance of high intensity intermittent exercise. It was hypothesized first that during the caffeine trial time to exhaustion would increase, and secondarily that (1) rating of perceived exertion would decrease, (2) blood lactate levels would increase, and (3) the respiratory exchange ratio would decrease when compared to the placebo trial.

Ventilation volume and heart rate were explored to determine if there were any differences between the caffeine and placebo trials.

Repeated measures ANOVA was used to determine any significant differences between the caffeine and placebo trials for any of the dependent variables (time to exhaustion, RPE, blood lactate, RER, VE, heart rate). Correlation and linear regression were used to further define the relationships and trends identified. There were no significant differences of the effect of caffeine between males and females on any of the variables measured, as shown by two factor repeated measures ANOVA. 


\subsubsection{Participant Characteristics}

Data for two of the original ten female participants were deleted prior to data analysis due to unusual values for time to exhaustion during one of their experimental trials. One of the participants who was sick on the day of the test chose to complete the test but performed poorly. During the fourth sprint to exhaustion she ran for 14.75 seconds, which was 8.25 seconds shorter than her final sprint during the habituation trial. The other participant stopped running after 19.39 seconds during one experimental trial compared with 35.46 seconds during the habituation trial, and she reported a RPE of 13 (somewhat hard). When asked why she got off the treadmill she stated that she could have run longer but didn't feel like it. Since neither participant was willing to repeat the trial on another occasion these participants were excluded from the study.

\subsubsection{Time to Exhaustion}

A two way repeated measures ANOVA (gender x treatment) showed no significant differences in time to exhaustion, $\mathrm{F}(1,15)=2.457, p=0.138$. Although not significant, the mean was higher $(51.58 \pm 23.77$ seconds $)$ during the caffeine trial than during the placebo trial (49.58 \pm 21.57 seconds), shown in Figure 3.1. 


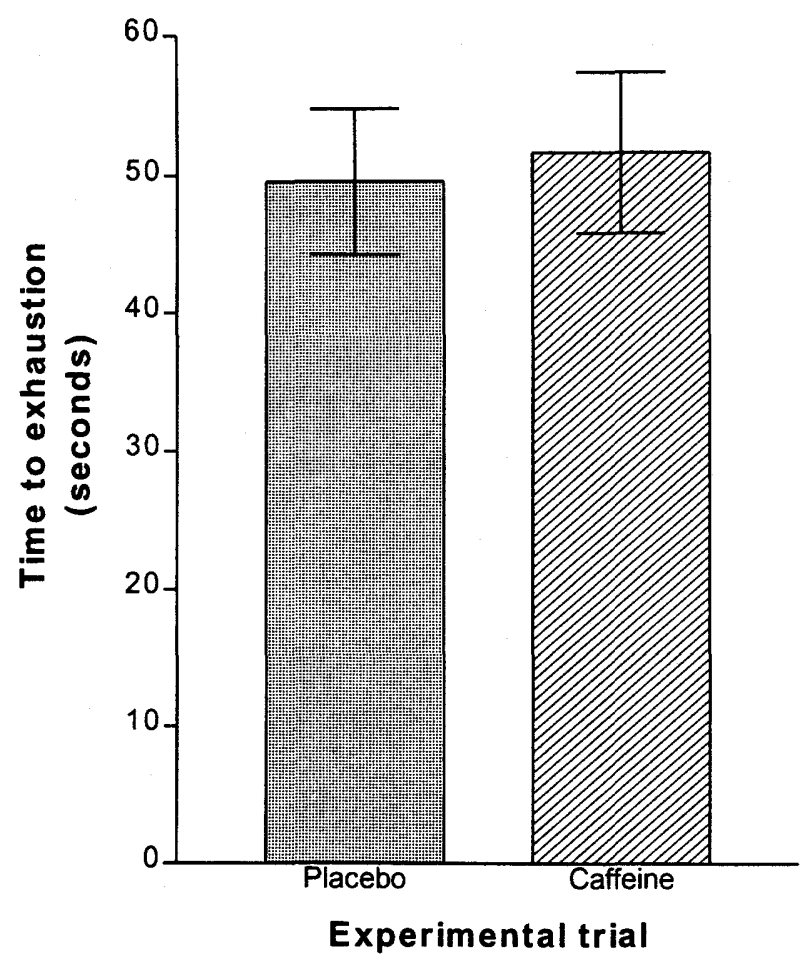

Placebo trial:

Mean $=49.58$

SEM $=5.24$

Caffeine trial:

Mean $=\mathbf{5 1 . 5 8}$

SEM $=5.77$

Figure 3.1. Mean time to exhaustion ( \pm standard error of the mean) during the experimental trials in seventeen male and female subjects

Time to exhaustion increased by $4.0 \%$ from the placebo to the caffeine trial for the whole sample, by $3.3 \%$ in the female participants, and by $4.3 \%$ in the male participants.

Correlations were performed between rating of perceived exertion, blood lactate, ventilation volume, heart rate, and the respiratory exchange ratio during the third sprint and time to exhaustion during the fourth sprint. This was done to determine whether the physiological variables during the sprint immediately prior to the final sprint had any impact on the time taken to run to exhaustion. All were negatively correlated with time to exhaustion during both experimental trials. RPE $(r=-0.61)$, RER $(r=-0.54)$, and heart rate $(r=-0.56)$ were significantly correlated with time to exhaustion during the placebo trial and RPE $(r=-0.58)$, blood lactate $(r=-0.75)$, and VE $(r=-0.50)$ were significantly 
correlated with time to exhaustion during the caffeine trial. Correlations among the variables during the placebo trial are presented in Table 3.1. Correlations for the caffeine trial are presented in Table 3.2 .

Table 3.1

Pearson correlations for seventeen participants during the placebo trial

\begin{tabular}{|c|c|c|c|c|c|c|}
\hline & 1 & 2 & 3 & 4 & 5 & 6 \\
\hline 1. Time to exhaustion & -- & $-.61 * *$ & -.44 & -.19 & $-.56^{*}$ & $-.54 *$ \\
\hline 2. RPE & & -- & $.67 * *$ & .38 & .42 & $.58 *$ \\
\hline 3. Blood lactate & & & -- & .07 & $.63 * *$ & .39 \\
\hline 4. Ventilation volume & & & & -- & .09 & .23 \\
\hline 5. Heart rate & & & & & -- & .24 \\
\hline 6. RER & & & & & & -- \\
\hline
\end{tabular}

Note. RPE, blood lactate, VE, heart rate, and RER are the values obtained during the third sprint. Time to exhaustion was obtained during the fourth sprint.

$* p<.05, * * p<.01$. 
Table 3.2

Pearson correlations for seventeen participants during the caffeine trial

\begin{tabular}{|c|c|c|c|c|c|c|}
\hline & 1 & 2 & 3 & 4 & 5 & 6 \\
\hline 1. Time to exhaustion & -- & $-.58^{*}$ & $-.75^{* *}$ & $-.50 *$ & -.41 & -.47 \\
\hline 2. RPE & & -- & $.65^{* *}$ & .40 & .08 & $.59 *$ \\
\hline 3. Blood lactate & & & -- & .31 & .24 & $.57^{*}$ \\
\hline 4. Ventilation volume & & & & -- & .10 & .22 \\
\hline 5. Heart rate & & & & & -- & .02 \\
\hline 6. RER & & & & & & -- \\
\hline
\end{tabular}

Note. RPE, blood lactate, VE, heart rate, and RER are the values obtained during the third sprint. Time to exhaustion was obtained during the fourth sprint.

$* p<.05, * * p<.01$.

During observation of the individual results it was determined that more

participants had a longer time to exhaustion during the caffeine trial than those who did not. Five out of the eight female participants ran longer during the caffeine trial, as

shown in Figure 3.3, and six out of the nine male participants ran longer with caffeine, as shown in Figure 3.4. In all, eleven of the seventeen participants improved their time to exhaustion during the caffeine trial. A Friedman's non-parametric test showed that this trend towards a longer time to exhaustion during the caffeine trial was not statistically significant, $X^{2}(1)=1.471, p=0.225$. 


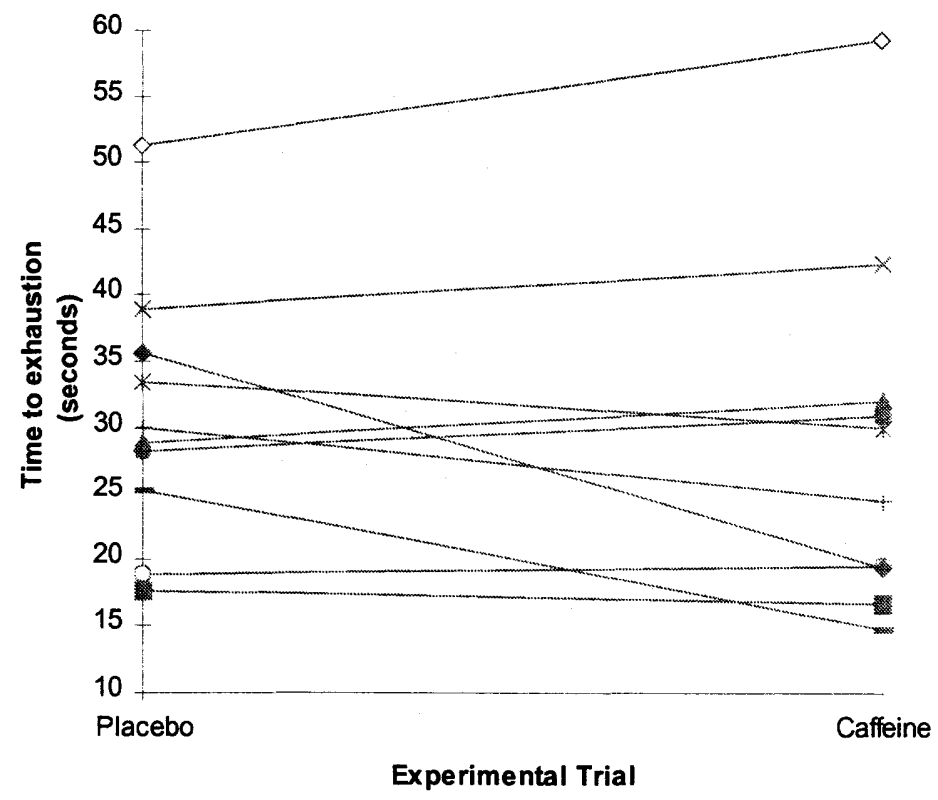

Figure 3.2. Individual time to exhaustion values during the experimental trials in eight female subjects.

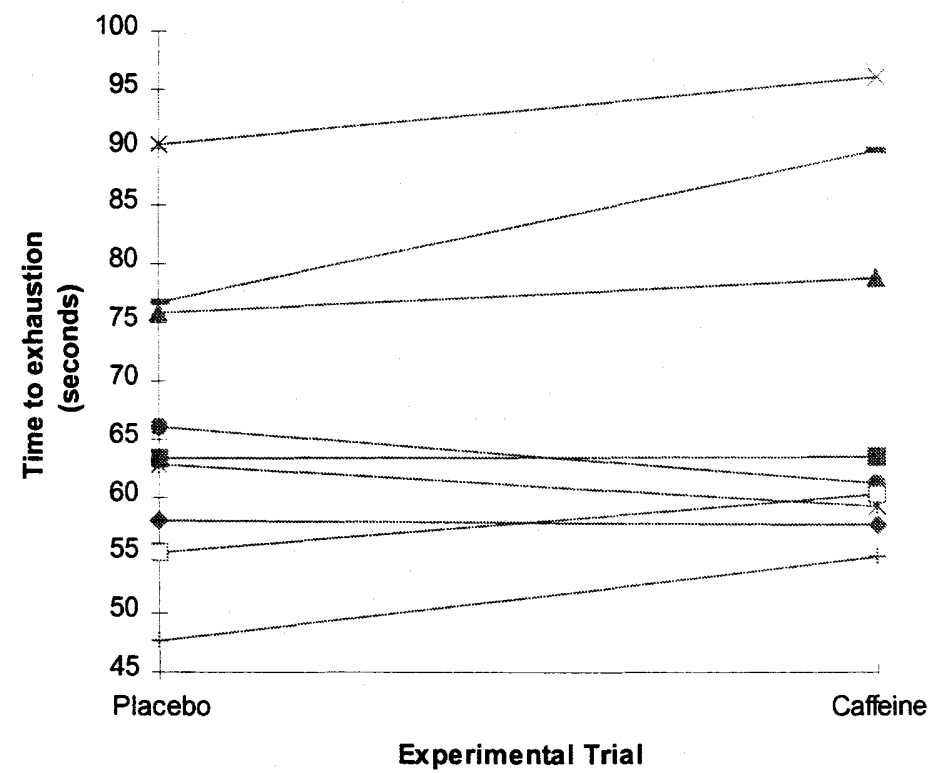

Figure 3.3. Individual time to exhaustion values during the experimental trials in nine male subjects. 


\subsubsection{Rating of Perceived Exertion}

A two factor repeated measures ANOVA (treatment $x$ sprint) showed no effect of caffeine on RPE during the first three sprints, $\mathrm{F}(1,16)=2.930, p=0.106$. Repeated measures ANOVA showed no significant differences in RPE between the caffeine and placebo trials for the sprint to exhaustion, $\mathrm{F}(1,16)=1.704, p=0.210$. The RPE data are presented in Table 3.3.

Table 3.3

Values of rating of perceived exertion (mean \pm standard deviation) during the experimental trials for seventeen subjects

\begin{tabular}{ccc}
\hline Sprint & $\begin{array}{c}\text { Placebo trial } \\
\text { (Borg RPE scale) }\end{array}$ & $\begin{array}{c}\text { Caffeine trial } \\
\text { (Borg RPE scale) }\end{array}$ \\
\hline 1 & $13.0 \pm 1.5$ & $12.4 \pm 2.0$ \\
2 & $14.2 \pm 1.8$ & $13.8 \pm 2.1$ \\
3 & $15.3 \pm 2.4$ & $15.1 \pm 2.3$ \\
4 & $18.2 \pm 1.6$ & $17.8 \pm 1.9$
\end{tabular}

Note. No significant differences between the trials.

\subsubsection{Blood Lactate}

Repeated measures ANOVA showed no significant differences between the caffeine and placebo trials on any of the three blood lactate measurement occasions taken. The test statistic was $\mathrm{F}(1,16)=0.621, p=0.442$ for the third sprint, $\mathrm{F}(1,15)=$ $0.444, p=0.515$ for the fourth sprint, and $\mathrm{F}(1,16)=1.060, p=0.319$ for the recovery measurement. The blood lactate data are presented in Table 3.4. 
Table 3.4

Blood lactate values (mean \pm standard deviation) during the experimental trials for seventeen subjects

\begin{tabular}{lcc}
\hline Blood lactate sample & $\begin{array}{c}\text { Placebo trial } \\
(\mathrm{mmol} / \mathrm{L})\end{array}$ & $\begin{array}{c}\text { Caffeine trial } \\
(\mathrm{mmol} / \mathrm{L})\end{array}$ \\
\hline Sprint 3 & $7.18 \pm 1.78$ & $7.55 \pm 2.00$ \\
Sprint 4 & $8.43 \pm 2.57$ & $8.93 \pm 2.46$ \\
Recovery 4 & $9.91 \pm 1.93$ & $10.54 \pm 2.51$
\end{tabular}

Note. No significant differences between the trials.

\subsubsection{Respiratory Factors}

A two factor repeated measures ANOVA (treatment x sprint) showed no effect of caffeine on the respiratory exchange ratio during the first three sprints, $\mathrm{F}(1,16)=0.023$, $p=0.882$. Repeated measures ANOVA showed no significant differences in RER between the caffeine and placebo trials during the 0 to 30 second time period of the fourth sprint, $F(1,16)=0.457, p=0.509$, nor the 31 to 60 second time period, $F(1,10)$ $=3.981, p=0.074$. During the 61 to 90 second time period RER was significantly lower during the caffeine trial, $\mathrm{F}(1,2)=100.00, p=0.010$. However, there were only three male subjects who ran long enough to have values in this time range. RER data are presented in Table 3.5. 
Table 3.5

Respiratory exchange ratio values (mean \pm standard deviation) during the experimental trials for seventeen subjects

\begin{tabular}{lcc}
\hline RER & Placebo trial & Caffeine trial \\
\hline Baseline & $0.963 \pm 0.073$ & $0.932 \pm 0.094$ \\
Sprint 1 & $0.848 \pm 0.072$ & $0.843 \pm 0.069$ \\
Recovery 1 & $1.146 \pm 0.070$ & $1.125 \pm 0.090$ \\
Sprint 2 & $0.915 \pm 0.057$ & $0.912 \pm 0.091$ \\
Recovery 2 & $1.121 \pm 0.078$ & $1.136 \pm 0.083$ \\
Sprint 3 & $0.878 \pm 0.048$ & $0.893 \pm 0.118$ \\
Recovery 3 & $1.082 \pm 0.060$ & $1.104 \pm 0.066$ \\
Sprint $4: 0-30$ seconds & $0.905 \pm 0.070$ & $0.893 \pm 0.084$ \\
Sprint $4: 31-60$ seconds & $0.970 \pm 0.066$ & $0.938 \pm 0.045$ \\
Sprint $4: 61-90$ seconds & $1.169 \pm 0.053$ & $1.137 \pm 0.064 * *$ \\
Recovery 4 & $1.052 \pm 0.067$ & $1.059 \pm 0.059$ \\
\hline$* * 0.01$, significant & & \\
\hline
\end{tabular}

$*^{*} p<0.01$, significant difference between the placebo and caffeine trials using repeated measures ANOVA.

Repeated measures ANOVA also indicated a significant difference in ventilation volume during the second $(\mathrm{F}(1,16)=4.651, p=0.047)$ and third $(\mathrm{F}(1,16)=6.933, p=$ $0.018)$ sprints, and during the final recovery period $(\mathrm{F}(1,16)=7.832, p=0.013)$, as shown in Table 3.6. 
Table 3.6

Ventilation volume values (mean \pm standard deviation) during the experimental trials for seventeen subjects

\begin{tabular}{lcc}
\hline Ventilation volume & $\begin{array}{c}\text { Placebo trial } \\
(\mathrm{L} / \mathrm{min})\end{array}$ & $\begin{array}{c}\text { Caffeine trial } \\
(\mathrm{L} / \mathrm{min})\end{array}$ \\
\hline Baseline & $17.07 \pm 3.62$ & $19.22 \pm 4.24$ \\
Sprint 1 & $68.03 \pm 11.56$ & $70.73 \pm 11.52$ \\
Recovery 1 & $68.76 \pm 8.57$ & $70.46 \pm 11.35$ \\
Sprint 2 & $95.36 \pm 10.34$ & $98.98 \pm 11.76^{*}$ \\
Recovery 2 & $74.13 \pm 9.04$ & $76.55 \pm 11.89$ \\
Sprint 3 & $101.51 \pm 12.65$ & $106.30 \pm 10.74^{*}$ \\
Recovery 3 & $75.31 \pm 8.18$ & $81.12 \pm 13.72$ \\
Sprint $4: 0-30$ seconds & $106.27 \pm 12.18$ & $110.53 \pm 14.33$ \\
Sprint $4: 31-60$ seconds & $140.18 \pm 11.49$ & $139.95 \pm 10.65$ \\
Sprint $4: 61-90$ seconds & $138.82 \pm 14.16$ & $147.02 \pm 19.80$ \\
Recovery 4 & $61.81 \pm 9.40$ & $69.37 \pm 9.93^{*}$
\end{tabular}

${ }^{*} p<0.05$, significant difference between the placebo and caffeine trials using repeated measures ANOVA.

\subsubsection{Heart Rate}

A two factor repeated measures ANOVA (treatment $\mathrm{x}$ sprint) showed no effect of caffeine on heart rate during the first three sprints, $\mathrm{F}(1,16)=2.145, p=0.162$. Repeated measures ANOVA showed no significant differences in heart rate during the fourth sprint between the experimental trials: $\mathrm{F}(1,16)=0.139, p=0.714$ from 0 to 30 seconds, $\mathrm{F}(1$, $10)=0.304, p=0.593$ from 31 to 60 seconds, and $F(1,1)=1.000, p=0.500$ from 61 to 90 seconds. Heart rate data are presented in Table 3.7. 
Table 3.7

Heart rate values (mean \pm standard deviation) during the experimental trials for seventeen subjects

\begin{tabular}{lcc}
\hline Heart rate & $\begin{array}{c}\text { Placebo trial } \\
\text { (beats/min) }\end{array}$ & $\begin{array}{c}\text { Caffeine trial } \\
\text { (beats/min) }\end{array}$ \\
\hline Baseline & $97.6 \pm 14.9$ & $100.8 \pm 15.9$ \\
Sprint 1 & $159.4 \pm 24.2$ & $166.4 \pm 13.1$ \\
Recovery 1 & $141.5 \pm 21.0$ & $142.9 \pm 22.0$ \\
Sprint 2 & $172.1 \pm 11.2$ & $174.9 \pm 11.5$ \\
Recovery 2 & $149.7 \pm 18.7$ & $150.4 \pm 20.0$ \\
Sprint 3 & $175.0 \pm 11.2$ & $176.4 \pm 10.0$ \\
Recovery 3 & $153.9 \pm 15.9$ & $154.1 \pm 17.3$ \\
Sprint 4: $0-30$ seconds & $176.4 \pm 8.3$ & $175.6 \pm 11.2$ \\
Sprint 4: $31-60$ seconds & $187.4 \pm 8.4$ & $188.2 \pm 8.7$ \\
Sprint 4: $61-90$ seconds & $187.8 \pm 3.9$ & $190.3 \pm 9.0$ \\
Recovery 4 & $136.2 \pm 14.7$ & $137.1 \pm 17.2$
\end{tabular}

Note. No significant differences between trials.

\subsubsection{Moderating Variables}

Repeated measures ANCOVA showed no significant differences when habitual caffeine consumption $(\mathrm{F}(1,14)=0.238, p=0.633)$ and physical activity levels $(\mathrm{F}(1,14)$ $=1.062, p=0.320$ ) were entered as covariates, nor were any of the covariates significant. These variables had no significant effect on the differences in performance between the caffeine and placebo trials. Repeated measures ANOVA showed that the composition of diets did not differ significantly between the placebo and caffeine trials (Table 3.8). 
Table 3.8

Dietary variables (mean \pm standard deviation) for the three days prior to the experimental trial for seventeen participants

\begin{tabular}{lcc}
\hline & Placebo trial & Caffeine trial \\
\hline Kilojoules / day & $9556.7 \pm 2724.0$ & $9168.3 \pm 2666.2$ \\
\% carbohydrate & $50.8 \pm 8.7$ & $53.1 \pm 5.6$ \\
$\%$ protein & $16.5 \pm 3.0$ & $16.6 \pm 3.3$ \\
$\%$ fat & $28.7 \pm 6.1$ & $28.9 \pm 4.2$ \\
\hline
\end{tabular}

Note. No significant differences between trials.

\subsection{Discussion}

The purpose of this study was to investigate the effect of caffeine on high intensity intermittent exercise performance and rating of perceived exertion, blood lactate, respiratory exchange ratio, ventilation volume, and heart rate during exercise. Seventeen subjects ( 9 male, 8 female) each performed two experimental trials following double-blind ingestion of caffeine or placebo. Participants completed three 30 second sprints on the treadmill each separated by two minutes of walking, followed by a fourth sprint to exhaustion.

Previous research on the effect of caffeine on high intensity exercise performance suggests that caffeine improves performance variables such as time to exhaustion (Doherty, 1998; Jackman et al., 1996; Trice \& Haymes, 1995). All three studies used time to exhaustion as the performance variable and found significant improvements in performance during the caffeine trial as compared to the placebo trial. These studies provided the basis for the proposed hypothesis that time to exhaustion would increase 
during the caffeine trial during this study. However, results from this study did not support this hypothesis. Nevertheless, the data appeared to indicate a trend towards an increase in time to exhaustion during the caffeine trial. Further analysis using a Friedman's non-parametric test indicated this trend to be non-significant.

Performance increased an average of $4.0 \%$, which is lower than the increases seen previously of $14.0 \%$ (Doherty, 1998), 19.7\% (Jackman et al., 1996), and 29.0\% (Trice \& Haymes, 1995). Trice \& Haymes (1995) alternated one minute of cycling with one minute of rest for 30 minutes and repeated this up to three times, to exhaustion. Jackman et al. (1996) used two 2 minute cycling periods separated by 6 minutes, followed by a cycle to exhaustion. Both of these studies with intermittent exercise used longer work periods than those used in this study, which may have accounted for the discrepancies between the results. In the other studies, time to exhaustion ranged from three minutes (Doherty, 1998) to five minutes (Jackman et al., 1996) to seventy-seven minutes (Trice \& Haymes, 1995). The time to exhaustion in this study ranged from seventeen to ninety-six seconds, which was much shorter than that of the other studies, making glycogen sparing as a mechanism for the ergogenic effect of caffeine less likely. However, total exercise time from the beginning of the first sprint to the end of the final sprint ranged from eight to nine minutes, which did fall within the time range of the other studies.

Prior to testing, the number of participants necessary to find a significant difference with $80 \%$ power was calculated (Glasnapp \& Poggio, 1985). This was done based on findings of previous research that used time to exhaustion as the performance variable during high intensity intermittent exercise (Jackman et al., 1996; Trice \& Haymes, 1995). Since the exercise time to exhaustion in this study was shorter than that 
found in earlier studies, the absolute differences seen were smaller. It is possible that this study did not include enough participants to show statistically significant changes in time, and if more participants had been used there may have been enough power to demonstrate significant differences between the placebo and caffeine trials.

It is important to mention that although the changes found were small and statistically non-significant, they may be all that is required to give athletes that extra edge needed in a field setting. With extremely narrow margins of difference between elite level athletes, a $4 \%$ improvement in performance may make a large difference in performance outcome. During the 1998 Winter Olympics in Nagano, Japan, there was a $3.8 \%$ difference (2:04.0 minutes compared to 2:08.9 minutes) between the top twelve finishers in the men's giant slalom snowboarding. As well, there was a $3.8 \%$ difference (1:16.6 minutes compared to 1:19.6 minutes) between the top sixteen finishers in the women's $500 \mathrm{~m}$ speed skating.

Correlations between time to exhaustion during the fourth sprint and all of the dependent variables measured during the third 30 second sprint were negative for both the caffeine and placebo trials. Since RPE, blood lactate, RER, heart rate, and VE all provide an indication of how hard the individual is working, negative correlations were expected, although they were not all significant. The lower these values are the easier the exercise is for that individual, and he or she should be able to run longer during the sprint to exhaustion. A lower RPE following the third sprint would suggest that the participant ought to run longer during the fourth sprint. The lower the RPE value is, the farther the participant is from exhaustion, so he or she would be expected to perform for a longer 
period of time before reaching exhaustion during the subsequent sprint. The same relationship was seen for blood lactate, VE, heart rate, and RER.

It is interesting to note that the significant correlations between time to exhaustion during the fourth sprint and RPE, blood lactate, VE, heart rate, and RER during the third sprint differed between the placebo trial and caffeine trials. Both trials had a significant correlation between time to exhaustion and RPE $(r=-.61$, placebo trial and $r=-.58$, caffeine trial). During the placebo trial, heart rate $(r=-0.56)$ and RER $(r=-0.54)$ were also significantly correlated with time to exhaustion, whereas during the caffeine trial, blood lactate $(r=-0.75)$ and VE $(r=-0.50)$ were significantly correlated. This would suggest that the effect of caffeine on high intensity exercise performance may be mediated by factors that affect blood lactate and VE. One potential mediating factor would be an increase in the circulating catecholamines, epinephrine and norepinephrine, which have been shown to increase following caffeine ingestion (Nehlig et al., 1992). Epinephrine and norepinephrine both stimulate ventilation as well as increase glycogenolysis (Fox, 1993), increasing the availability of glucose for glycolysis, resulting in an increased concentration of muscle and blood lactate. During the caffeine trial, blood lactate during the third sprint was significantly correlated with time to exhaustion during the fourth sprint. This may indicate that during this trial, when subjects were closer to being physiologically fatigued, they jumped off the treadmill due to a build-up of lactate in their blood and muscles causing an increase in hydrogen ion concentration. This suggests that the tendency towards the improvement in performance observed during the caffeine trial may be due to caffeine's effect on the central nervous system (Nehlig et al., 1992). Caffeine increases $\beta$-endorphins and serotonin levels in the body 
(Nehlig et al., 1992; Spiller, 1998), both of which are involved in the sensation of pain (Fox, 1993). $\beta$-endorphins increase the pain threshold and are associated with feelings of euphoria and analgesia (Fox, 1993) and serotonin modulates pain perception (Fox, 1993). Increasing the pain threshold may postpone the sensations associated with physical exertion that cause the perception of exhaustion.

Individual analysis of the data shows that the majority of the subjects increased their time to exhaustion during the caffeine trial, when compared to the placebo trial (Figures 3.2 and 3.3). Six out of the nine males and five of eight females improved their performance (11 out of 17). Since repeated measures ANCOVA showed that the covariate habitual caffeine consumption was not significant, this cannot be considered when explaining the individual variations. However, it is possible that individual differences in the sensitivity to caffeine exist which could account for the differences seen. It has already been suggested that it is extremely difficult to differentiate between habituation to the effects of caffeine and variations in sensitivity to these effects (Nehlig et al., 1992). The results from the individual data analysis suggest that some individuals are more susceptible to the ergogenic effects of caffeine than others. Eleven out of the seventeen participants improved their performance independent of their regular exercise patterns, normal caffeine consumption, or diet suggesting that some other variable caused this individualized response. None of these variables were significant covariates, and no clear patterns or trends could be identified using the group means or the individual data. Research to date does not allow for the prediction of which athletes will benefit from the ergogenic properties of caffeine and which ones will not. 
One of the potential limitations with this study is that the athletes may not have been trained enough to demonstrate the ergogenic effects of caffeine, which seems to depend on the training status of the individual (Collomp et al., 1991; Collomp et al., 1992). Although these subjects were athletes competing at the university level, this does not necessarily mean or ensure that they are highly trained. Athletes who would most benefit from very small improvements in performance would be those who were sufficiently trained to be able to compete at national and international levels. Such athletes would also be more likely to provide consistent performances between trials, making it more likely that any differences seen between trials would more likely be due to the effect of caffeine.

Measuring the athletes in their post-competitive season, as occurred in this study, might also have been a limitation since most individuals' structured training had decreased by this time (March to May). Therefore, the participants in this study were not necessarily tested when they were in peak physical condition. Two previous studies measured $\mathrm{VO}_{2 \max }$ prior to the experimental trials and demonstrated $\mathrm{VO}_{2 \max }$ values of 60.0 $\pm 4.5 \mathrm{~mL} / \mathrm{kg} / \mathrm{min}$ (Doherty, 1998) and $54.5 \pm 15.2 \mathrm{~mL} / \mathrm{kg} / \mathrm{min}$ (Trice \& Haymes, 1995). From these studies, a minimum $\mathrm{VO}_{2 \max }$ value of approximately $50 \mathrm{~mL} / \mathrm{kg} / \mathrm{min}$ would be recommended in order to demonstrate the ergogenic properties of caffeine.

Another potential limitation may have been the use of both male and female subjects. The uniform treadmill speed of $7.5 \mathrm{mph}$ was too difficult for some of the female participants. Two were unable to run for the required 30 seconds during the third sprint. Therefore, these subjects would have pushed themselves to exhaustion during the third sprint before undergoing the sprint to exhaustion. In addition, many of the female 
participants ran for less than 30 seconds during the fourth sprint, which is less than the 30 to 60 seconds range recommended by the original Cunnigham and Faulkner (1969) protocol. The Pearson-Product correlation between the time to exhaustion values during the habituation and placebo trials provides an indication of the test-retest reliability of this protocol. The modified Cunnigham and Faulkner (1969) treadmill test provided highly reproducible performances $(r=0.976, \mathrm{p}=0.000)$.

A learning component may have been involved from trial to trial, which may have affected the results. The accustomization and habituation trials were designed to familiarize the participants with the procedures in order to minimize the chances of learning effects becoming a factor. However, twelve out of the seventeen participants increased time to exhaustion from their first to their second experimental trial. Four of these subjects received the placebo on the second visit. Only three out of seven participants who received caffeine at the first experimental visit increased time to exhaustion with the caffeine, compared to eight out of ten participants who received caffeine at the second experimental trial. This suggests that learning may have had a greater effect on performance than caffeine in some of the participants, indicating that more than one habituation trial may have been necessary to minimize the effects of learning.

Several studies that have examined RPE during high intensity exercise have found no significant differences in RPE despite improvements in performance (MacIntosh \& Wright, 1995; Trice \& Haymes, 1995; Wiles et al., 1992). These studies suggested that athletes perceived themselves to be working at a similar intensity during both the caffeine and placebo trials, when they were actually working at a higher intensity during the 
caffeine trial (MacIntosh \& Wright, 1995). Results from this study did not find any significant differences in RPE between the caffeine and placebo trials, but the RPE values were on average lower during the caffeine trial following the sprints. There was no expected change in RPE following the fourth sprint between the caffeine and placebo trials, since the subjects were running to voluntary exhaustion. Therefore, the expected result was that subjects would run to the same RPE in each trial, but would run longer following caffeine ingestion. However, during the first three sprints it was expected that the RPE values would be lower during the caffeine trials, since the subjects were working at the exact same intensity for these sprints during both trials, which did not occur. It was expected that the caffeine ingestion would cause the participants to perceive themselves to be exercising at a lower intensity. It is possible that the Borg scale (Borg, 1982) does not provide small enough intervals to detect changes as small as those caused by caffeine during this study for the first three sprints.

It was hypothesized that blood lactate would be increased during the caffeine trial as compared to the placebo trial. This would indicate greater utilization of energy from glycolysis, which would be expected if the subjects ran for a longer period of time. Previously, Doherty (1998), Trice and Haymes (1995), and Jackman et al. (1996) have all found no significant changes in blood lactate following high intensity exercise. It is possible that this might reflect an increase in lipolysis rather than glycolysis to supply the additional energy for the improvement in performance. In the current study, repeated measures ANOVA did not show any significant differences between trials, however blood lactate increased non-significantly during the caffeine trial. This suggests that 
anaerobic glycolysis, rather than lipolysis, thus producing more lactate, provided any additional energy needed for the small improvements in performance.

Increased glycolysis seemed to be the source of additional energy for all but three males who demonstrated decreases in RER. RER can be used to estimate substrate utilization for energy where a lower ratio indicates a greater percentage of fat oxidation (Williams, 1995a). It was hypothesized that RER would be lower during the caffeine trial, suggesting an increase in lipolysis. The results showed no differences between the trials except in the subjects who ran from 61 to 90 seconds during the final sprint. The RER was significantly lower during the caffeine trial in these three male subjects. This suggests that an increase in lipolysis may be involved in improved performance following caffeine ingestion during high intensity exercise lasting longer than one minute.

The increase in VE observed during the second and third sprints and the final recovery period during the caffeine trial does not support the results from previous studies (Berry et al., 1991; Doherty, 1998). It is possible that the increased VE was due to the need to expire excess carbon dioxide levels caused by an increase in blood lactate. Increased carbon dioxide levels stimulate the chemoreceptors that trigger an increase in ventilation volume (Wilmore \& Costill, 1994).

In conclusion, the ingestion of caffeine prior to high intensity intermittent exercise seems to improve performance by a narrow and statistically non-significant margin. The effect of caffeine on exercise performance appears to be individualized, however neither habitual caffeine consumption, normal physical activity levels, nor food intake were able to predict which athletes might be susceptible to the ergogenic benefits of caffeine. 
Caffeine did not significantly increase time to exhaustion although eleven out of seventeen participants did demonstrate an increase. 


\section{CHAPTER 4}

\section{SUMMARY AND CONCLUSIONS}

\subsection{Summary}

In recent years, sports have become more publicized and analyzed. A large number of research studies are being performed to determine how to maximize exercise performance coincident with this popularity of sports. Caffeine is one of the many substances that athletes may ingest in an effort to optimize their performance (Lamarine, 1998). Caffeine has been shown to improve performance during endurance exercise (Tarnopolsky, 1994), but its effects on high intensity intermittent exercise are uncertain.

This study was performed to determine the effects of acute caffeine ingestion on high intensity intermittent exercise performance with respect to rating of perceived exertion, blood lactate, respiratory exchange ratio, ventilation volume, and heart rate. It was hypothesized that caffeine ingestion would cause an increase in time to exhaustion, lower RPE, increase blood lactate, and decrease RER.

Seventeen college level athletes ( 9 male, 8 female) underwent caffeine and placebo intermittent, high intensity exercise trials in a double-blind randomized order. Habitual caffeine consumption, normal physical activity levels, and food intake for three days prior to each trial were assessed by questionnaires. One hour before beginning 
exercise, subjects were given $6 \mathrm{mg} / \mathrm{kg}$ body weight of either caffeine or placebo (lactose) rounded to the nearest $100 \mathrm{mg}$ (average dose of $511 \mathrm{mg}$ for males and $430 \mathrm{mg}$ for females). The exercise portion included three 30 second sprints on the treadmill at $7.5 \mathrm{mph}$ and a $20 \%$ incline each separated by two minutes of walking. A fourth and final sprint to exhaustion followed this, where time to exhaustion was measured as the performance variable. At the end of each sprint, subjects reported their RPE. Blood lactate was measured following the third and fourth sprints and after five minutes of recovery. Respiratory variables $\left(\mathrm{VCO}_{2}, \mathrm{VO}_{2}, \mathrm{VE}\right)$ were measured throughout the testing protocol and the RER was calculated.

Repeated measures ANOVA did not show any significant differences between the caffeine and placebo trials for time to exhaustion, RPE, blood lactate, or RER. These results do not support any of the hypotheses. However, the mean values for the placebo and caffeine trials demonstrated patterns in the expected directions.

Time to exhaustion increased by an average of $4.0 \%$ during the caffeine trial and eleven out of the seventeen subjects ran longer following the ingestion of caffeine. Caffeine may improve performance by acting on the central nervous system and altering the perception of physical discomfort, allowing participants to perform longer.

RPE was lower after all sprints and blood lactate was higher at all collection times during the caffeine versus the placebo trials. This suggests that the athletes perceived themselves to be working at a lower intensity while they were actually working at least at an identical intensity during the caffeine trial. Increased blood lactate levels indicate an increase in anaerobic glycolysis, which may be providing the additional energy needed to perform for a longer time period. However, three males had a significantly lower RER 
during the caffeine trial for the 61 to 90 second time period of the sprint until exhaustion. This lower RER represents a greater utilization of free fatty acids for energy so lipolysis may be supplying the additional fuel for this extended time period.

Although this study did not show any significant ergogenic effects of caffeine on high intensity intermittent exercise performance, the conditions under which caffeine would be beneficial need to be considered. At high levels of competition, a $4 \%$ improvement in exercise performance could give an athlete the advantage he or she needs to become a champion.

\subsection{Conclusions}

According to the results from this study, the following conclusions can be made:

1. Caffeine ingestion did not significantly increase time to exhaustion.

2. Caffeine ingestion did not significantly decrease rating of perceived exertion.

3. Caffeine ingestion did not significantly increase blood lactate levels.

4. Caffeine ingestion did not appear to decrease respiratory exchange ratio, except from 61 to 90 seconds of the fourth sprint, indicating an increase in lipolysis during this time period.

5. Caffeine ingestion increased ventilation volume during the second and third sprints and during the final recovery period.

6. Caffeine ingestion had no effect on heart rate.

7. Habitual caffeine consumption and normal physical activity levels did not explain the variability in the results. 


\subsection{Recommendations for Future Research}

This study monitored the training status of the participants, their habitual caffeine consumption, and their diet immediately prior to the testing trials. These factors have previously been suggested to potentially explain the variability seen in performance results following caffeine ingestion (Collomp et al., 1991; Doherty, 1998; MacIntosh \& Wright, 1995; Trice \& Haymes, 1995). Despite assessing these mediating variables, they were unable to explain the differences in response to caffeine observed among the participants. In the future, the participant characteristics should be more tightly controlled. Based on the findings from this study, the following are recommendations for future research:

1. Use elite athletes who compete at the national or international level where such small changes in performance may have large consequences in outcome. In addition, elite athletes would be more likely to provide consistent performances and therefore any changes in performance could be attributed more confidently to caffeine.

2. Evaluate athletes during their competitive season when they are in peak condition rather than the post season when structured training has decreased.

3. Evaluate participants of one gender so that a testing protocol can be selected where all participants would reach exhaustion in approximately 60 seconds, since caffeine seems to provide ergogenic benefit during exercise of slightly longer time intervals.

4. Assess fitness levels initially to ensure that all subjects are highly trained individuals.

5. Include two or more habituation trials (until performance plateaus) to ensure that order effects are not involved. 
6. Include a true control group that performs the same number of trials as the experimental group without taking either caffeine or placebo capsules to determine whether a placebo effect exists.

7. Assess other blood factors, such as free fatty acids, epinephrine, and norepinephrine, to gain more insight into the mechanisms involved in the ergogenic properties of caffeine. 


\section{REFERENCES}

Anselme, F., Collomp, K., Mercier, B., Ahmaïdi, S., \& Préfaut, Ch. (1992). Caffeine increases maximal anaerobic power and blood lactate concentration. European Journal of Applied Physiology and Occupational Physiology, 65, 188-191.

Bangsbo, J., Jacobsen, K., Nordberg, N., Christensen, N. J., \& Graham, T. (1992). Acute and habitual caffeine ingestion and metabolic responses to steady-state exercise. Journal of Applied Physiology, 72, 1297-1303.

Berglund, B. \& Hemmingsson, P. (1982). Effects of caffeine ingestion on exercise performance at low and high altitudes in cross-country skiers. International Journal of Sports Medicine, 3, 234-236.

Berry, M. J., Stoneman, J. V., Weyrich, A. S., \& Burney, B. (1991). Dissociation of the ventilatory and lactate thresholds following caffeine ingestion. Medicine and Science in Sports and Exercise, 23, 463-469.

Borg, G. A. V. (1982). Psychophysical bases of perceived exertion. Medicine and Science in Sports and Exercise, 14, 377-381.

Bouchard, C., Taylor, A. W., Simoneau, J.-A., \& Dulac, S. (1991). Testing anaerobic power and capacity. In J. D. MacDougall, H. A. Wenger, \& H. J. Green (Eds.), Physiological Testing of the High-Performance Athlete ( $2^{\text {nd }}$ ed.), (pp. 175-221). Champaign, IL: Human Kinetics.

Bucci, L. (1993). Nutrients as Ergogenic Aids for Sports and Exercise. Boca Raton, FL: CRC Press.

Bucci, L. R. (1994). Nutritional ergogenic aids. In I. Wolinsky \& J. F. Hickson, Jr. (Eds.), Nutrition in Exercise and Sport (2nd ed.), (pp. 295-346). Boca Raton, FL: CRC Press.

Burke, L. \& Heeley, P. (1994). Dietary supplements and nutritional ergogenic aids in sport. In L. Burke \& V. Deakin (Eds.), Clinical Sports Nutrition, (pp. 227-284). New York: McGraw-Hill.

Cadarette, B. S., Levine, L., Berube, C. L., Posner, B. M., \& Evans, W. J. (1983). Effects of varied dosages of caffeine on endurance exercise to fatigue. In H. G. Knuttgen, J. A. Vogel, \& J. Poortmans (Eds.), Biochemistry of Exercise, (pp. 871-876).

Champaign, IL: Human Kinetics.

Canadian Society for Exercise Physiology. (1996). Professional Fitness and Lifestyle Consultant: Resource Manual. Ottawa, Canada: Author. 
Clarkson, P. M. (1993). Nutritional ergogenic aids: caffeine. International Journal of Sport Nutrition, 3, 103-111.

Collomp, K., Ahmaïdi, S., Audran, M., Chanal, J.-L., \& Préfaut, Ch. (1991). Effects of caffeine ingestion on performance and anaerobic metabolism during the Wingate test. International Journal of Sports Medicine, 12, 439-443.

Collomp, K., Ahmaïdi, S., Chatard, J. C., Audran, M., \& Préfaut, Ch. (1992). Benefits of caffeine ingestion on sprint performance in trained and untrained swimmers. European Journal of Applied Physiology and Occupational Physiology, 64, 377-380.

Costill, D. L., Dalsky, G. P., \& Fink, W. J. (1978). Effects of caffeine ingestion on metabolism and exercise performance. Medicine and Science in Sports, 10, 155-158.

Cunningham, D. A. \& Faulkner, J. A. (1969). The effect of training on aerobic and anaerobic metabolism during a short exhaustive run. Medicine and Science in Sports, 1 , 65-69.

Dodd, S. L., Brooks, E., Powers, S. K., \& Tulley, R. (1991). The effects of caffeine on graded exercise performance in caffeine naive versus habituated subjects. European Journal of Applied Physiology and Occupational Physiology, 62, 424-429.

Doherty, M. (1998). The effects of caffeine on the maximal accumulated oxygen deficit and short-term running performance. International Journal of Sport Nutrition, 8, 95-104.

Engels, H.-J. \& Haymes, E. M. (1992). Effects of caffeine ingestion on metabolic responses to prolonged walking in sedentary males. International Journal of Sport Nutrition, 2, 386-396.

Fox, S. I. (1993). Human Physiology (4 ${ }^{\text {th }}$ ed.). Dubuque, Iowa: Wm. C. Brown.

Glasnapp, D. R. \& Poggio, J. P. (1985). Essentials of Statistical Analysis for the Behavioral Sciences. Columbus, $\mathrm{OH}$ : Charles E. Merrill Publishing.

Godin, G. \& Shephard, R. J. (1985). A simple method to assess exercise behaviour in the community. Canadian Journal of Applied Sport Sciences, 10, 141-146.

Graham, T. E., Rush, J. W. E., \& van Soeren, M. H. (1994). Caffeine and exercise: metabolism and performance. Canadian Journal of Applied Physiology, 19, 111-138.

Graham, T. E. \& Spriet, L. L. (1991). Performance and metabolic responses to a high caffeine dose during prolonged exercise. Journal of Applied Physiology, 71, 2292-2298. 
Graham, T. E. \& Spriet, L. L. (1995). Metabolic, catecholamine, and exercise performance responses to various doses of caffeine. Journal of Applied Physiology, 78, 867-874.

Greer, F., McLean, C., \& Graham, T. E. (1998). Caffeine, performance, and metabolism during repeated Wingate exercise tests. Journal of Applied Physiology, 85, 1502-1508.

Haynes, S. P. \& Fitch, K. D. (1992). Doping. In J. Bloomfield, P. A. Fricker, \& K. D. Fitch (Eds.), Textbook of Science and Medicine in Sport, (pp. 525-534). Champaign, IL: Human Kinetics.

Ivy, J. L., Costill, D. L., Fink, W. J., \& Lower, R. W. (1979). Influence of caffeine and carbohydrate feedings on endurance performance. Medicine and Science in Sports, 11, 611.

Jackman, M., Wendling, P., Friars, D., \& Graham, T. E. (1996). Metabolic, catecholamine, and endurance responses to caffeine during intense exercise. Journal of Applied Physiology, 81, 1658-1663.

Kowalchuk, J. M., Heigenhauser, G. J. F., Lindinger, M. I., Obminski, G., Sutton, J. R., \& Jones, N. L. (1988). Role of lungs and inactive muscle in acid-base control after maximal exercise. Journal of Applied Physiology, 65, 2090-2096.

Lamarine, R. J. (1998). Caffeine as an ergogenic aid. In G. A. Spiller (Ed.), Caffeine, (pp. 233-250). New York: CRC Press.

Lindinger, M. I., Graham, T. E., \& Spriet, L. L. (1993). Caffeine attenuates the exerciseinduced increase in plasma $\left[\mathrm{K}^{+}\right]$in humans. Journal of Applied Physiology, 74, 1149 1155 .

Lundsberg, L. S. (1998). Caffeine consumption. In G. A. Spiller (Ed.), Caffeine, (pp. 199-224). New York: CRC Press.

MacIntosh, B. R. \& Wright, B. M. (1995). Caffeine ingestion and performance of a 1,500-metre swim. Canadian Journal of Applied Physiology, 20, 168-177.

McArdle, W. D., Katch, F. I., \& Katch, V. L. (1991). Exercise Physiology: Energy, Nutrition, and Human Performance (3rd ed.). Philadelphia: Lea \& Febiger.

Nehlig, A., Daval, J.-L., \& Debry, G. (1992). Caffeine and the central nervous system: mechanisms of action, biochemical, metabolic and psychostimulant effects. Brain Research Reviews, 17, 139-170. 
Pasman, W. J., van Baak, M. A., Jeukendrup, A. E., \& de Haan, A. (1995). The effect of different dosages of caffeine on endurance performance time. International Journal of Sports Medicine, 16, 225-230.

Sasaki, H., Maeda, J., Usui, S., \& Ishiko, T. (1987). Effect of sucrose and caffeine ingestion on performance of prolonged strenuous running. International Journal of Sports Medicine, 8, 261-265.

Sensormedics Corporation. (1995). Vmax Series Reference Manual. U.S.A.: Author.

Smith, B. D. \& Tola, K. (1998). Caffeine: effects on psychological functioning and performance. In G. A. Spiller (Ed.), Caffeine, (pp. 251-299). New York: CRC Press.

Spiller, G. A. (1998). Basic metabolism and physiological effects of the methylxanthines. In G. A. Spiller (Ed.), Caffeine, (pp. 225-231). New York: CRC Press.

Spriet, L. L., MacLean, D. A., Dyck, D. J., Hultman, E., Cederblad, G., \& Graham, T. E. (1992). Caffeine ingestion and muscle metabolism during prolonged exercise in humans. American Journal of Physiology, 262, E891-E898.

Stone, M. H. \& Conley, M. S. (1994). Bioenergetics. In T. R. Baechle (Ed.), Essentials of Strength Training and Conditioning, (pp. 67-85). Champaign, IL: Human Kinetics.

Tarka, S. M., Jr. \& Hurst, W. J. (1998). Introduction to the chemistry, isolation, and biosynthesis of methylxanthines. In G. A. Spiller (Ed.), Caffeine, (pp. 1-11). New York: CRC Press.

Tarnopolsky, L. J., MacDougall, J. D., Atkinson, S. A., Tarnopolsky, M. A., \& Sutton, J. R. (1990). Gender differences in substrate for endurance exercise. Journal of Applied Physiology, 68, 302-308.

Tarnopolsky, M. A. (1994). Caffeine and endurance performance. Sports Medicine, 18, 109-125.

Trice, I. \& Haymes, E. M. (1995). Effects of caffeine ingestion on exercise-induced changes during high-intensity, intermittent exercise. International Journal of Sport Nutrition, 5, 37-44.

Vander, A. J., Sherman, J. H., \& Luciano, D. S. (1990). Human Physiology: The Mechanisms of Body Function ( $5^{\text {th }}$ ed.). New York: McGraw-Hill.

Van Soeren, M. H., Sathasivam, P., Spriet, L. L., \& Graham, T. E. (1993). Caffeine metabolism and epinephrine responses during exercise in users and nonusers. Journal of Applied Physiology, 75, 805-812. 
Wiles, J. D., Bird, S. R., Hopkins, J., \& Riley, M. (1992). Effect of caffeinated coffee on running speed, respiratory factors, blood lactate and perceived exertion during $1500-\mathrm{m}$ treadmill running. British Journal of Sports Medicine, 26, 116-120.

Williams, J. H. (1991). Caffeine, neuromuscular function and high-intensity exercise performance. Journal of Sports Medicine and Physical Fitness, 31, 481-489.

Williams, J. H., Signorile, J. F., Barnes, W. S., \& Henrich, T. W. (1988). Caffeine, maximal power output and fatigue. British Journal of Sports Medicine, 22, 132-134.

Williams, M. H. (1995a). Nutrition for Fitness and Sport (4th ed.). Toronto, Canada: Brown \& Benchmark.

Williams, M. H. (1995b). Nutritional ergogenics in athletics. Journal of Sports Sciences, 13, S63-S74.

Wilmore, J. H. \& Costill, D. L. (1994). Physiology of Sport and Exercise. Champaign, IL: Human Kinetics.

Yellow Springs Instrument Company. (1991). Model 2300 Stat Glucose and L-Lactate Analyzer Operation Manual. Yellow Springs, OH: Author. 
APPENDICES 


\section{Appendix A Consent Form}




\section{Title of Project:}

\section{Effect of acute caffeine ingestion on high intensity intermittent exercise performance in elite male and female athletes}

Principal Investigator: D.T. Drinkwater, Ph.D., College of Kinesiology, University of Saskatchewan, Tel: 966-6468

Co-Investigator: H.K. Whelan, Graduate Student, College of Kinesiology, University of Saskatchewan, Tel: 966-2688

The purpose of this study is to determine if caffeine ingestion improves performance during high intensity, intermittent exercise.

You will be required to attend the laboratory on four separate occasions. The first time will be in a group and will be to become accustomed to running on the treadmill at the speed and incline to be used during testing. The second visit will be a habituation procedure. You will go through the testing procedures exactly as you would during the testing trials. The final two visits will be the testing trials. One will be the caffeine trial and the other the placebo trial. Neither you nor the researcher will know which one you have taken. The dose of caffeine that will be given is $6 \mathrm{mg}$ caffeine per kg body weight. One cup of coffee contains approximately 100-150 mg of caffeine. The placebo will be an equivalent amount of lactose. You must sit and wait for one hour after taking the caffeine or placebo to allow for its distribution throughout the body.

You will warm up on the treadmill for 5 minutes and rest and stretch for 5 minutes. The treadmill will be set at a $20 \%$ grade and at a speed of $7.5 \mathrm{mph}$. You must sprint for 30 seconds at this speed three times. Each sprint will be separated by 2 minutes of fast walking on the treadmill. A fourth and final sprint will be run until exhaustion and you can no longer continue. Following this final sprint, there will be a 5-minute recovery period where you will walk on the treadmill.

Throughout the protocol, you will be wearing a facemask hooked up to the metabolic cart. This will measure various factors of your breathing. Immediately upon the completion of all of the sprints you will have to rate how exerted you felt on the Borg scale of perceived exertion. Your blood lactate will be measured immediately following the final 30-second sprint, the sprint to exhaustion, and after the 5-minute recovery period. Blood will be taken by a finger prick and only one prick should be necessary on each testing occasion.

If you choose to participate, you will be free to withdraw from the study at any time without fear of any penalty. Your individual results are confidential and will only be seen by the research team. Your identity will not be revealed and the final report will summarize the group results. The findings of this project will be made available to you upon your request at the end of the project. 


\section{YOUR STATEMENT OF CONSENT}

Project Title: Effect of acute caffeine ingestion on high intensity intermittent exercise performance in elite male and female athletes

Investigators: D.T. Drinkwater, Ph.D. (966-6468) and H.K. Whelan, B.Sc. (966-2688)

I have received explanations about the nature of the study, its purpose, and procedures. I understand what my participation in this study will involve.

I am a volunteer and can withdraw at any time from the study without any fear of penalty.

I understand that I will be required to come to the laboratory on four separate occasions. The first time will last approximately 45 minutes, the second time approximately 1 hour and the final two times approximately 2 hours each.

I will be required to run repeated sprints on the treadmill, which may cause muscle soreness for 1-2 days after the tests.

I will have a small amount of blood taken by a finger prick on each testing day.

On one occasion I will be given a dose of caffeine corresponding to my body weight and a lactose placebo pill on the other. I will not know which one I am being given.

The individual data that I provide will remain confidential from sources outside of the study. My identity will not be revealed in reports and research publications.

I will receive a summary of the project, upon request, following the completion of the project.

Signature of Participant

Date

Signature of Witness

Date

Signature of Investigator

Date 
Appendix B Three-Day Food Record 
College of Physical Education

University of Saskatchewan

THREE-DAY FOOD RECORD

NAME:

DATES:

SPORT:

AGE:

HEIGHT:

WEIGHT: 


\section{INTRODUCTION}

This booklet is used to record your detailed daily food intake. It is meant to give the researchers some idea of your usual dietary intake. Therefore, it is very important that you do not alter your eating habits while taking part in this study. In other words, do not let the fact that you are writing down what you eat influence your choice of foods. The names of the participants in this study will be kept confidential.

The usefulness of the results of this study depends on the accuracy with which you record your daily food intake. Please write down full details on all the food and drink that you consume each day. 


\section{INSTRUCTIONS}

1) The purpose of this diary is to record all the food (including drinks) which you eat for a three day period. The three day period should include 2 weekdays and 1 weekend day.

2) Two pages are provided for each day of the three day period.

3) After each meal or snack that you eat, please write down in detail each separate food item you consumed - including the type of food (e.g. processed cheese) and the amount of food in household measures (e.g. 1 cup of cooked spaghetti). A meal will have to be listed by its separate parts (e.g. fried steak - 8 oz., french fries - 1 cup, coleslaw - 3 tbsp.)

4) The best way to record the information is by carrying this diary around with you wherever you go. Before going to sleep, you should look over the diary to check that you have not missed anything. Remember to include snacks!

5) If you eat fast food, you can just list the type of food you ate (e.g. $1 \mathrm{Big} \mathrm{Mac,} 1$ large fires, 1 chocolate milkshake).

6) The following pages explain the use of household measures, and the description of foods. A sample day's diet sheet is given. Please take the time to read these pages as it will help to make your diet record more accurate. 


\section{RECORDING IN THE DIARY}

1) Please use household measures. For example:

cup: vegetables, cereal, fruit, milk, beverages

tablespoon: sauces, fats

teaspoon: sugar, honey, drink mix

slices: bread, bacon

fractions: $1 / 6$ pie.

2) State the type of food eaten. For example:

Milk: homo, 2\%, $1 \%$, skim, goat's

Cheese: processed, Swiss, spread

Bread: enriched white, $60 \%$ whole wheat, sweet cinnamon bun, bran muffin

Cereal: Sugar Pops, Miniwheats, granola

Meat: hamburger, fried chicken - breasts, scrambled eggs, cod fillets

Others: $\quad$ strawberry jam, Becel margarine, Caesar dressing, oatmeal cookies.

3) State the amount of food eaten. For example:

Cheese: 1" cube cheddar

3 tbsp lite cream cheese

$1 / 4$ cup $2 \%$ creamed cottage cheese

Fruit: $1 / 2$ cup canned peaches (in heavy syrup)

12 grapes

1 medium banana

Bread: 2 slices $100 \%$ whole wheat

1 large kaiser

Cereal: $3 / 4$ cup corn flakes

1 shredded wheat biscuit 
Meat: 1 cup baked beans with pork

2 cups tuna casserole (tuna, cream of mushroom soup, noodles, peas)

4 thin slices roast beef

Vegetables: 2 slices cucumber

$1 / 2$ cup boiled cabbage

4) Include manner of cooking. fried, boiled, raw.

5) Remember all alcoholic drinks. 
Here is a Sample:

Date: Sat., Dec. 14th (Day 3)

\begin{tabular}{|c|c|c|c|}
\hline Time & Food Description & Amount & Code \\
\hline \multirow[t]{5}{*}{$9: 30 \mathrm{a} . \mathrm{m}$} & Waffles-white flour & $3,8 " \times 4 "$ & ea. \\
\hline & syrup-Aunt Jemima & $1 / 2$ cup & \\
\hline & yogurt-peach & $125 \mathrm{ml}$ & \\
\hline & coffee, 1 tsp. sugar & 1 cup & \\
\hline & $\operatorname{milk}(2 \%)$ & $1 / 4$ cup & \\
\hline \multirow[t]{3}{*}{$10: 30$} & Chocolate chip cookies & 3 & \\
\hline & coffee, 1 tsp. sugar & |l & \\
\hline & milk (Half \& Half-10\%) & $1 / 4$ cup & \\
\hline \multirow[t]{7}{*}{$12: 30$} & Sandwich & & \\
\hline & -2 slices whole wheat bread & 2 slices & \\
\hline & -mozarella cheese $\left(3^{\prime \prime} \times 1 / 4^{\prime \prime} \times 2^{\prime \prime}\right)$ & 2 slices & \\
\hline & salami & 4 slices & \\
\hline & - lettuce & 1 leaf & \\
\hline & butter & $1+\operatorname{sen}$ & \\
\hline & -mayonaise & 1 tsp. & \\
\hline \multirow[t]{5}{*}{$5: 30$} & Spaghetti & 1 cup & \\
\hline & meat sauce & $1 / 2$ cup & \\
\hline & garlic toast & 2 slices & \\
\hline & Continue on the next page if & your nee & \\
\hline & it) Leave Code column blank & & \\
\hline
\end{tabular}




\section{Date:}

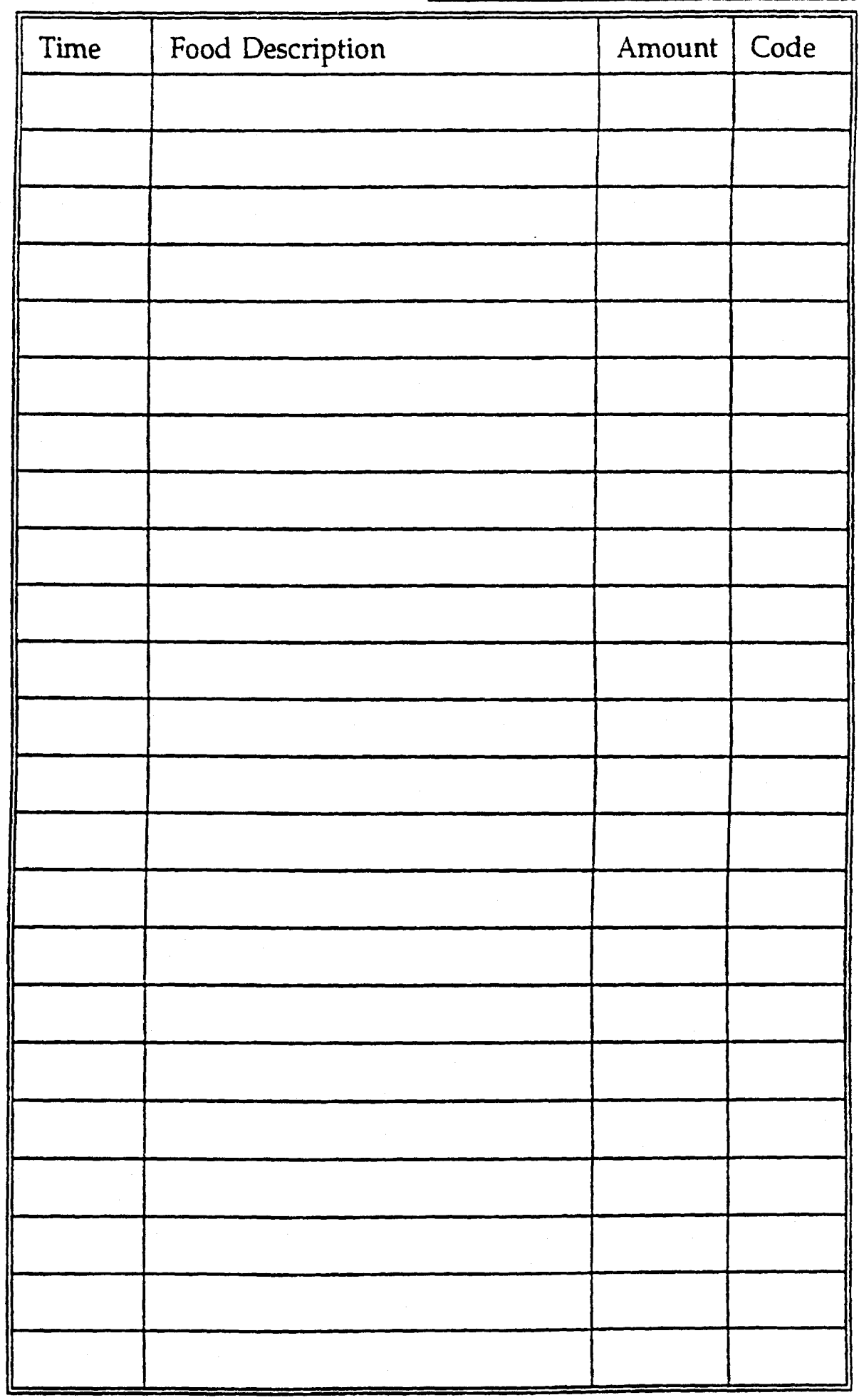


Appendix C Subject Instruction Form 


\section{Caffeine and High Intensity Intermittent Exercise Study \\ College of Physical Education, University of Saskatchewan}

\section{INSTRUCTIONS}

Please follow these instructions. If you don't follow any one of these items, you will not be allowed to perform the tests.

\section{NO CAFFEINE - 3 DAYS}

You may not eat or drink anything that contains caffeine for 72 hours before the tests.

Caffeine is found in coffee, tea, soft drinks, chocolate.

NO ANAEROBIC EXERCISE - 2 DAYS

You may not do any type of anaerobic exercise for 48 hours before the tests.

Anaerobic exercise includes weight training, interval training.

\section{NO AEROBIC EXERCISE - 1 DAY}

You may not do any type of aerobic exercise for 24 hours before the tests.

Aerobic exercise includes running, biking.

\section{NO FOOD - 2 HOURS}

You may not eat any food or drink anything other than water for 2 hours before the tests.

Please do not forget to bring your completed 3-day food diary with you on the date of the test.

Your trials have been scheduled at the following times:

Control: date:

time:

Trial \#1: date:

time:

Trial \#2: date:

time:

Someone will call you the evening before your tests to remind you. Please let us know if you are unable to come. 
Appendix D Data Recording Sheet 


\section{Caffeine and High Intensity Intermittent Exercise Study}

College of Physical Education, University of Saskatchewan

\section{DATA RECORDING}

Name:

Subject number:

Date:

Height: $\mathrm{cm}$

Weight: $\mathrm{kg}$

Rating of perceived exertion:

Sprint 1:

Sprint 2:

Sprint 3:

Sprint 4:

Time to exhaustion (sprint 4): seconds

Blood lactate:

Sprint 3:

Sprint 4:

5 minute recovery:

Preparation Checklist:

No caffeine - 72 hours

YES NO

No anaerobic exercise - 48 hours YES NO

No aerobic exercise - $\mathbf{2 4}$ hours YES NO

No food - 2 hours

YES NO

3 day food diary

YES NO

Drug Administration:

Time of drug:

Time of test start:

Dose of Drug: $\quad 6 \mathrm{mg} \times$ body weight $\mathbf{k g}=$ mg 
Appendix E Leisure Time Exercise Questionnaire 


\section{Caffeine and High Intensity Intermittent Exercise Study \\ College of Physical Education, University of Saskatchewan \\ LEISURE TIME EXERCISE QUESTIONNAIRE}

Name:

Subject number:

Date:

1. Considering a 7-day period (a week), how many times on average do you do the following kinds of exercise for more than 15 minutes during your free time (write in each circle the appropriate number)?

TIMES PER WEEK

a) STRENUOUS EXERCISE (HEART BEATS RAPIDLY)

(i.e. running, jogging, hockey, football, soccer, squash, basketball, cross country skiing, judo, roller skating, vigorous swimming, vigorous long distance bicycling)

b)

MODERATE EXERCISE
(NOT EXHAUSTING)

(i.e. fast walking, baseball, tennis, easy bicycling, volleyball, badminton, easy swimming, alpine skiing, popular and folk dancing)

c) MILD EXERCISE (MINIMAL EFFORT)

(i.e. yoga, archery, fishing from river bank, bowling, horseshoes, golf, snowmobiling, easy walking)
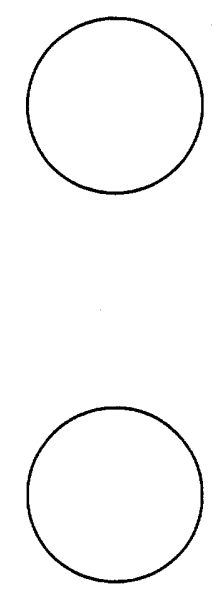

1. Considering a 7-day period (a week), during your leisure-time, how often do you engage in any regular activity long enough to work up a sweat (heart beats rapidly)?

OFTEN

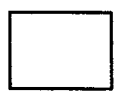

SOMETIMES

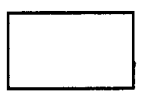

NEVER/RARELY

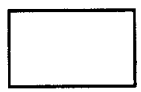


Appendix F Estimation of Habitual Caffeine Consumption Questionnaire 


\section{Caffeine and High Intensity Intermittent Exercise Study \\ College of Physical Education, University of Saskatchewan}

\section{ESTIMATION OF HABITUAL CAFFEINE CONSUMPTION}

Name:

Subject number:

Date:

Instructions:

Use the following list to estimate your average daily caffeine intake. Some of the items give you a range of caffeine content to select from. For example: if you drink brewed coffee using the drip method, you are asked to select an answer that falls between 60 and $180 \mathrm{mg}$ of caffeine per 5-oz. cup of coffee. While on the average brewed coffee using the drip method contains $115 \mathrm{mg}$ caffeine, some people prefer a stronger coffee (i.e. higher caffeine content) while others prefer a less strong coffee (i.e. lower caffeine content). In these instances, always select the number that you believe will best represent your habitual caffeine intake of that time.

Scoring:

Be sure to make any necessary mathematical adjustments in reporting the average daily caffeine intake value for a product. For example, if you usually consume one Diet Pepsi (caffeine content: $36 \mathrm{mg} / 12$-oz. serving) during the course of a week, the corrected average daily caffeine consumption would be $36 / 7=5.1 \mathrm{mg} /$ day. If you consume $4 \mathrm{Diet}$ Pepsi's per day, the correct value would be $4 \times 36=144 \mathrm{mg} /$ day. 
PRODUCT

Coffee: (5-oz. cup)

Decaffeinated, instant

Decaffeinated, brewed

Instant

Brewed, drip method

Brewed, percolated

Tea: (5-oz. cup)

Brewed, major brands

Brewed, imported brands

Instant

Iced (12-oz. glass)

Cocoa beverage: (5-oz. cup)

Chocolate milk beverage: (8-oz. drink)

Milk chocolate: (1-oz.)

(1 bar of size $3.75 \times 1.5 \times 0.25$ in.) 6

40

60

30

70

4

$2-20$

2-7

$1-15$

Dark chocolate, semisweet:

$$
\text { (1-oz. } 1 \text { bar) }
$$

20

5-35

Chocolate flavoured syrup:

(1-oz. 1.5 tbsp)

4

4 
Soft drinks: (12-oz. serving)

Coca-Cola, Diet Coke

Pepsi-Cola

38.4

Diet Pepsi, Pepsi Light $\quad 30.0$

Mountain Dew $\quad 54.0$

$\begin{array}{ll}\text { TAB } & 46.8\end{array}$

Dr. Pepper, Sugar-Free Dr. Pepper 39.6

$\begin{array}{ll}\text { RC Cola } & 36.0\end{array}$

Canada Dry $\quad 30.0$

Canada Dry Diet Cola $\quad 1.2$

Prescription Drugs:

Cafergot (for migraine headache) $\quad 100$

Fiorinal (for tension headache) $\quad 40$

Soma compound (pain relief) $\quad 32$

Darvon Compund (pain relief) $\quad 32.4$

Weight-Control Aids:

Codexin 200

Dex-A-Diet II 200

Dexatrim, Dexatrim Extra-Strength 200

Dietac capsules 200

Maximum strength Appredine $\quad 100$

$\begin{array}{ll}\text { Prolamine } & 140\end{array}$ 
Alertness tablets:

NoDoz

Vivarin

200

Analgesic/Pain Relief:

Anacin, Maximum strength Anacin 37

Excedrin

65

Midol

32.4

Vanquish

33

Diuretics:

Aqua-Ban $\quad 100$

Maximum strength Aqua-Ban Plus 200

Permathene H2 Off 200

Cold/Allergy Remedies:

Coryban-D capsules $\quad 30$

Dristan Decongestant tablets $\quad 16.2$

TOTAL: 
Appendix G Diet Analysis Print-Out 


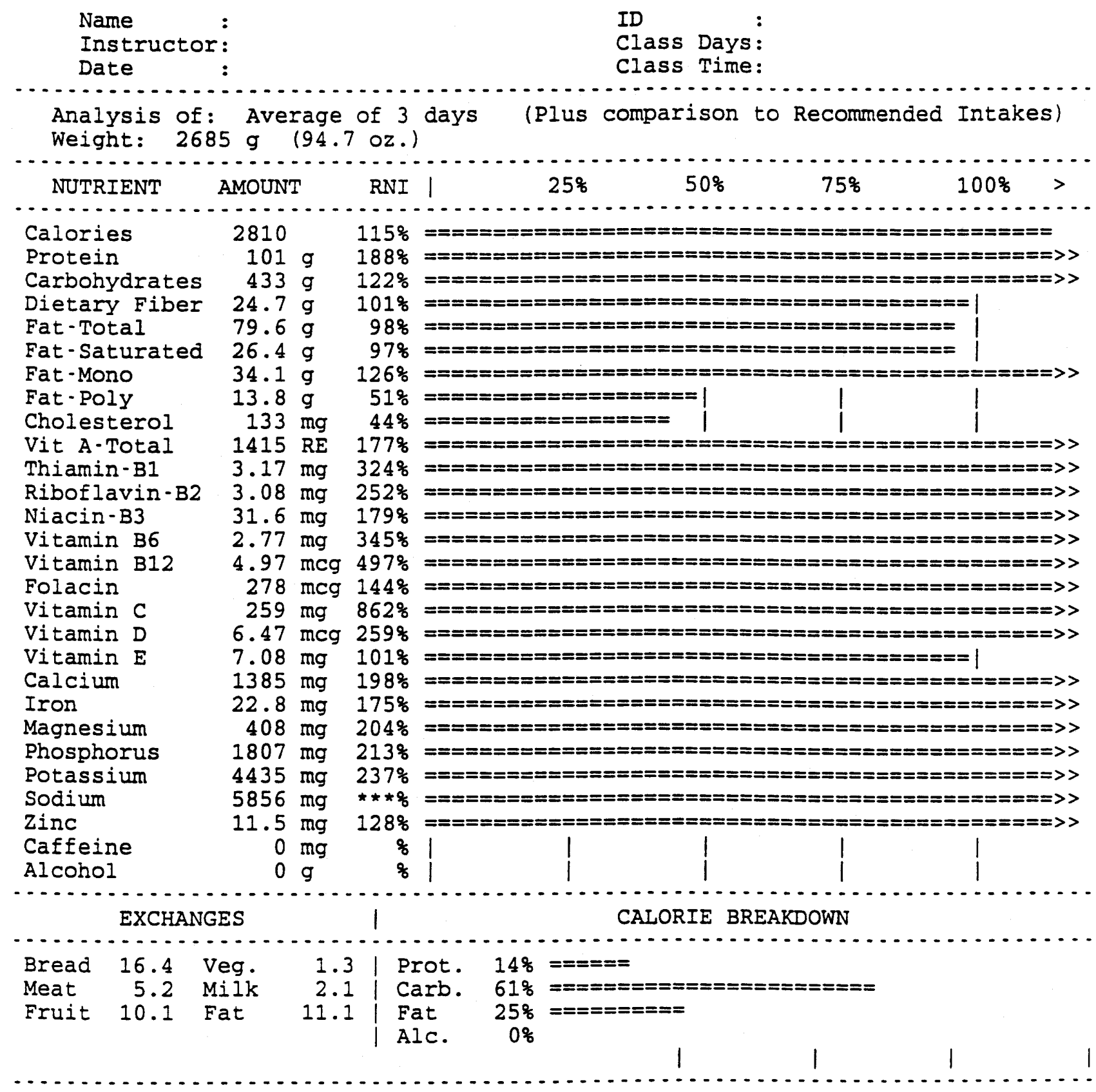


Appendix H PAR-Q (Physical Activity Readiness Questionnaire) 
Appendix I Background Information Form 


\section{Caffeine and High Intensity Intermittent Exercise Study}

College of Physical Education, University of Saskatchewan

\section{BACKGROUND INFORMATION}

Name:

Sport:

Birth date: day: month: year:

Gender: male: female:
Subject number:

Address:

Postal code:

Phone number:

Saskatchewan health insurance number:

\section{FOR OFFICE USE ONLY}

Treadmill orientation: date:

Habituation protocol: date:

Trial \#1:

Trial \#2:

Consent form signed:

PAR-Q signed:

Physical activity questionnaire:

Caffeine use questionnaire: date:

date:

NO

YES

NO

YES

NO

YES time:

time:

time: time: 
Appendix J Study Protocol

Visit 1

Visit 2, 3, 4

Sprinting Sequence

Drug Administration 


\section{Caffeine and High Intensity Intermittent Exercise Study \\ College of Physical Education, University of Saskatchewan}

\section{PROTOCOL}

\section{Visit 1}

1. Consent form, PAR-Q, and background information completely filled out and signed.

2. Fill out physical activity questionnaire and caffeine use questionnaire.

3. Warm up on bikes for 5 minutes.

4. Stretch for 5 minutes.

5. Put both treadmills at $20 \%$ incline and $7.5 \mathrm{mph}$.

6. Demonstrate how to get on:

a. Straddle treadmill and hold on to railing.

b. Put weight on handrails and get feet moving to the speed.

c. Once feet are going, let go of the handrails.

7. Demonstrate how to get off:

a. Grab onto handrails.

b. Put weight on handrails and lift feet off of the treadmill.

c. Straddle the treadmill.

8. Take turns running 30 second sprints on the treadmill.

9. Have everyone run at least one sprint until exhaustion.

10. Make sure that they bike in between sprints.

11. Give them the instruction sheet and book their 3 tests. 


\section{Caffeine and High Intensity Intermittent Exercise Study \\ College of Physical Education, University of Saskatchewan}

\section{PROTOCOL}

\section{Visit 2, 3, 4}

1. Fill out name and date on data recording sheet before subject arrives.

2. Assign a subject number.

3. Go through preparation checklist with subject (if they answer no to any question they are not allowed to be tested).

4. Check 3-day food diary to make sure that they have not had any caffeine.

5. Record height in $\mathrm{cm}$ and weight in $\mathrm{kg}$.

6. Figure out the dose of drug during visits 3 and 4 .

7. Give the data recording sheet to drug administrator during visits 3 and 4 .

8. Explain how the scale of rating of perceived exertion works.

9. Put on heart rate monitor and watch.

10. Warm-up on treadmill at $6 \mathrm{mph}$ and $0 \%$ incline (heart rate must reach $150 \mathrm{bpm}$ ).

11. Stretch for 5 minutes (heart rate must return to $100 \mathrm{bpm}$ )

12. Calibrate metabolic cart.

13. Put on facemask.

14. Hook up subject.

15. Collect baseline data for 5 minutes with subject standing by treadmill.

16. Follow the sprinting sequence protocol.

17. Double check the next appointment and give them the 3-day food diary. 


\section{Caffeine and High Intensity Intermittent Exercise Study \\ College of Physical Education, University of Saskatchewan \\ PROTOCOL}

\section{Sprinting Sequence}

1. Subject straddles treadmill while it is increased to $20 \%$ incline and $7.5 \mathrm{mph}$.

2. 30 second sprint

a. Start time when subject has their feet going.

b. Count down for them every 5 seconds.

3. Subject calls out their rating of perceived exertion.

4. Subject straddles treadmill while it is lowered to $5 \%$ incline and $3.5 \mathrm{mph}$.

5. Start timing 2 minutes at the end of the sprint and subject walks until the end.

6. Subject straddles treadmill while it is increased to $20 \%$ incline and $7.5 \mathrm{mph}$.

7. 30 second sprint

a. Start time when subject has their feet going.

b. Count down for them every 5 seconds.

8. Subject calls out their rating of perceived exertion.

9. Subject straddles treadmill while it is lowered to $5 \%$ incline and $3.5 \mathrm{mph}$.

10. Start timing 2 minutes at the end of the sprint and subject walks until the end.

11. Subject straddles treadmill while it is increased to $20 \%$ incline and $7.5 \mathrm{mph}$.

12. 30 second sprint

a. Start time when subject has their feet going.

b. Count down for them every 5 seconds.

13. Subject calls out their rating of perceived exertion. 
14. Subject straddles treadmill while it is lowered to $5 \%$ incline and $3.5 \mathrm{mph}$.

15. Prick finger and collect first blood sample.

16. Start timing 2 minutes at the end of the sprint and subject walks until the end.

17. Subject straddles treadmill while it is increased to $20 \%$ incline and $7.5 \mathrm{mph}$.

18. Subject sprints until exhaustion

a. Start time when they let go of the handrails (once they have their feet going).

b. Stop time when they grab back on.

c. Do not tell them what their time is, just tell them they are doing great.

19. Subject calls out their rating of perceived exertion.

20. Subject straddles treadmill while it is lowered to $0 \%$ incline and $3.5 \mathrm{mph}$.

21. Collect blood sample from open finger prick.

22. Cool-down for 5 minutes.

23. Collect final blood sample. 


\section{Caffeine and High Intensity Intermittent Exercise Study \\ College of Physical Education, University of Saskatchewan \\ PROTOCOL}

\section{Drug Administration}

1. Record the subject number and date in the double blind key.

2. Roll the die:
a. Even number $(2,4,6)=$ CAFFEINE
b. Odd number $(1,3,5)=$ PLACEBO

3. Record the roll of the die and the drug in the double blind key.

4. If it is their final visit, give them the drug they have not been given.

5. Double check the dose of drug calculation on the data recording sheet $(6 \mathrm{mg} / \mathrm{kg})$.

6. Round the dose to the nearest $100 \mathrm{mg}$.

7. Record the dose actually given in the double blind key.

8. Count out the capsules and give them to the subject with a glass of water.

9. Watch the subject take the drug.

10. Have the subject sit and wait for one hour. 


\section{Appendix K Metabolic Cart Print-Out}


College of Physical Education

University of Saskatchewan

Fitness Appraisal Lab

Saskatoon, Saskatchewan

Date:

Metabolic Edit -

Time Sec

HH:MM
Test Stage - Baseline

00:00:10

00:00:20

00:00:30

00:00:40

00:00:50

00:01:00

00:01:10

00:01:20

00:01:30

00:01:40

00:01:50

00:02:00

00:02:10

00:02:20

00:02:30

00:02:40

00:02:50

00:03:00

00:03:10

00:03:20

00:03:30

00:03:40

00:03:50

00:04:00

00:04:10

00:04:20

00:04:30

00:04:40

00:04:50

00:05:00

00:05:10

00:05:20

00:05:30

00:05:40

Test Stage - Exercise

00:00:10

00:00:20

00:00:30

00:00:40

Test Stage -Recovery
$\begin{array}{llll}H R & V E(B T P S) & V C O 2 & V O 2 \\ \mathrm{VO} & \mathrm{V} 2 / \mathrm{kg}\end{array}$ BPM

\section{7}

85

89

95

90

86

86

89

95

88

90

85

87

90

79

83

87

82

80

97

108

108

111

107

114

114

115

115

113

111

113

115

107

119

146

170

176

177

168

00:00:20

00:00:30

00:00:40

00:00:50

00:01:00

00:01:10

00:01:20

00:01:30

00:01:40

00:01:50

00:02:00
164

159

156

153

148

146

144

146

144

144

139
L/min

$\mathrm{L}$ min

$\mathrm{L} / \mathrm{min} \mathrm{mL} / \mathrm{kg} / \mathrm{min}$

22.3

0.619

0.484

0.446

0.504

0.449

0.423

0.489

0.369

0.448

0.234

0.309

0.283

0.387

0.244

0.516

0.817

0.147

0.478

0.518

0.638

0.791

0.968

0.995

0.980

0.999

0.929

1.030

0.915

0.925

0.839

0.794

0.823

0.785

26.4

52.0

1.612

2.248

3.419

2.745

72.7

78.4

93.5

92.6

3.221

3.746

3.567

105.4

3.665

3.234

3.232

2.758

2.790

2.378

2.707

2.188

1.963
0.618

\subsection{7}

0.468

0.431

0.502

0.433

0.403

0.467

0.351

0.448

0.212

0.309

0.289

0.626

0.342

0.236

0.538

0.775

0.130

0.506

0.548

0.703

0.835

1.003

1.032

0.990

1.008

0.943

1.036

0.968

0.932

0.893

0.824

0.806

0.769

1.556

2.458

4.005

2.908

3.120

3.432

3.079

2.934

2.644

2.651

2.226

2.430

2.093

2.388

1.895

1.672

109

$\begin{array}{rlll}7.1 & 1.00 & 17.49 & 3.57 \\ 5.3 & 1.04 & 17.66 & 3.53 \\ 4.9 & 1.04 & 17.73 & 3.45 \\ 5.7 & 1.03 & 17.80 & 3.35 \\ 4.9 & 1.04 & 17.83 & 3.35 \\ 4.6 & 1.05 & 17.83 & 3.37 \\ 5.3 & 1.06 & 17.78 & 3.41 \\ 4.0 & 1.06 & 17.72 & 3.49 \\ 5.1 & 1.03 & 18.06 & 3.07 \\ 2.4 & 1.12 & 18.55 & 2.71 \\ 3.5 & 1.00 & 17.54 & 3.42 \\ 3.3 & 1.00 & 18.08 & 2.98 \\ 7.1 & 1.03 & 17.76 & 3.34 \\ 3.9 & 1.14 & 18.50 & 2.86 \\ 2.7 & 1.07 & 18.64 & 2.54 \\ 6.1 & 0.97 & 17.41 & 3.58 \\ 8.8 & 1.04 & 17.55 & 3.66 \\ 1.5 & 1.16 & 19.03 & 2.23 \\ 5.7 & 0.94 & 17.19 & 3.75 \\ 6.2 & 0.94 & 17.48 & 3.47 \\ 8.0 & 0.91 & 16.86 & 3.96 \\ 9.5 & 0.95 & 16.90 & 4.02 \\ 11.4 & 0.97 & 16.84 & 4.15 \\ 11.7 & 0.97 & 16.67 & 4.31 \\ 11.2 & 0.99 & 16.77 & 4.30 \\ 11.5 & 0.99 & 16.67 & 4.40 \\ 10.7 & 0.99 & 16.73 & 4.32 \\ 11.8 & 1.00 & 16.78 & 4.30 \\ 11.0 & 0.95 & 16.55 & 4.37 \\ 10.6 & 0.99 & 16.80 & 4.29 \\ 10.1 & 0.94 & 16.96 & 3.96 \\ 9.4 & 0.96 & 16.78 & 4.18 \\ 9.2 & 1.03 & 17.07 & 4.11 \\ 8.7 & 1.03 & 17.21 & 3.97\end{array}$

17.7

27.9

45.5

33.0

1.03

0.93

0.85

0.94

35.5

39.0

35.0

33.3

30.0

30.1

25.3

27.6

23.8

27.1

21.5

19.0

1.03

1.09

1.18

1.25

1.22

1.22

1.24

1.15

1.13

1.13

1.16

1.18

17.13

16.52

16.24

16.15

4.09

4.30

4.32

4.74

15.84

16.18

16.65

5.40

5.27

5.03

4.59

17.06

4.68

4.69

4.50

4.54

4.56

16.93

16.97

4.52

4.47

17.25 
Date:

Metabolic Edit -

\begin{tabular}{|c|c|c|c|c|c|c|c|c|}
\hline $\begin{array}{r}\text { Time Sec } \\
\text { HH:MM } \\
00: 02: 10\end{array}$ & $\begin{array}{r}\text { HR } \\
\text { BPM } \\
134\end{array}$ & $\begin{array}{r}\text { VE(BTPS) } \\
\text { Umin } \\
52.6\end{array}$ & $\begin{array}{l}\mathrm{VCO} 2 \\
\mathrm{~L} / \mathrm{min} \\
1.684\end{array}$ & $\begin{array}{l}\text { VO2 } \\
L / \min \\
1.411\end{array}$ & $\begin{array}{r}\mathrm{VO} 2 / \mathrm{kg} \\
\mathrm{mL} / \mathrm{kg} / \mathrm{min} \\
16.0\end{array}$ & 1.19 & $\begin{array}{r}\text { FEO2 } \\
\% \\
17.39\end{array}$ & $\begin{array}{r}\text { FECO2 } \\
\% \\
4.24\end{array}$ \\
\hline \multicolumn{9}{|c|}{ Test Stage - Exercise } \\
\hline $00: 00: 10$ & 145 & 73.5 & 2.442 & 2.138 & 24.3 & 1.15 & 17.14 & 4.41 \\
\hline $00: 00: 20$ & 166 & 99.8 & 3.100 & 3.135 & 35.6 & 1.00 & 16.98 & 4.12 \\
\hline $00: 00: 30$ & 178 & 116.0 & 3.399 & 4.143 & 47.1 & 0.82 & 16.58 & 3.89 \\
\hline $00: 00: 40$ & 182 & 95.6 & 2.827 & 3.243 & 36.9 & 0.89 & 16.82 & 3.88 \\
\hline \multicolumn{9}{|c|}{ Test Stage -Recovery } \\
\hline 00:00:10 & 179 & 92.8 & 3.344 & 3.605 & 41.0 & 0.93 & 16.08 & 4.75 \\
\hline 00:00:20 & 173 & 92.9 & 3.386 & 3.524 & 40.0 & 0.96 & 16.15 & 4.81 \\
\hline 00:00:30 & 169 & 103.0 & 3.715 & 3.647 & 41.4 & 1.02 & 16.37 & 4.80 \\
\hline $00: 00: 40$ & 166 & 102.5 & 3.509 & 3.133 & 35.6 & 1.12 & 16.95 & 4.52 \\
\hline $00: 00: 50$ & 163 & 95.7 & 3.252 & 2.792 & 31.7 & 1.16 & 17.08 & 4.50 \\
\hline 00:01:00 & 159 & 97.3 & 3.295 & 2.849 & 32.4 & 1.16 & 17.10 & 4.46 \\
\hline $00: 01: 10$ & 157 & 84.0 & 2.807 & 2.435 & 27.7 & 1.15 & 17.13 & 4.40 \\
\hline 00:01:20 & 157 & 79.2 & 2.625 & 2.361 & 26.8 & 1.11 & 17.07 & 4.34 \\
\hline 00:01:30 & 154 & 76.7 & 2.543 & 2.245 & 25.5 & 1.12 & 16.97 & 4.47 \\
\hline 00:01:40 & 151 & 79.6 & 2.691 & 2.540 & 28.9 & 1.06 & 16.80 & 4.49 \\
\hline 00:01:50 & 151 & 73.4 & 2.396 & 2.200 & 25.0 & 1.09 & 17.07 & 4.28 \\
\hline 00:02:00 & 151 & 66.8 & 2.048 & 1.829 & 20.8 & 1.12 & 17.37 & 4.05 \\
\hline 00:02:10 & 148 & 57.7 & 1.813 & 1.593 & 18.1 & 1.14 & 17.30 & 4.17 \\
\hline 00:02:20 & 149 & 65.4 & 2.020 & 1.745 & 19.8 & 1.16 & 17.43 & 4.08 \\
\hline \multicolumn{9}{|c|}{ Test Stage - Exercise } \\
\hline 00:00:10 & 169 & 102.5 & 2.972 & 2.775 & 31.5 & 1.08 & 17.44 & 3.87 \\
\hline 00:00:20 & 181 & 124.8 & 3.288 & 3.943 & 44.8 & 0.84 & 17.05 & 3.53 \\
\hline 00:00:30 & 188 & 127.6 & 3.443 & 4.223 & 48.0 & 0.82 & 16.85 & 3.61 \\
\hline 00:00:40 & 189 & 94.7 & 2.872 & 3.286 & 37.3 & 0.87 & 16.64 & 4.01 \\
\hline \multicolumn{9}{|c|}{ Test Stage -Recovery } \\
\hline 00:00:10 & 184 & 93.3 & 3.023 & 3.261 & 37.1 & 0.93 & 16.55 & 4.30 \\
\hline 00:00:20 & 181 & 104.2 & 3.495 & 3.713 & 42.2 & 0.94 & 16.46 & 4.44 \\
\hline 00:00:30 & 175 & 98.8 & 3.257 & 3.183 & 36.2 & 1.02 & 16.83 & 4.36 \\
\hline $00: 00: 40$ & 171 & 91.6 & 3.115 & 2.910 & 33.1 & 1.07 & 16.84 & 4.47 \\
\hline $00: 00: 50$ & 169 & 95.5 & 3.182 & 2.889 & 32.8 & 1.10 & 16.96 & 4.41 \\
\hline 00:01:00 & 168 & 77.8 & 2.486 & 2.327 & 26.4 & 1.06 & 17.10 & 4.17 \\
\hline $00: 01: 10$ & 165 & 90.7 & 2.812 & 2.511 & 28.5 & 1.12 & 17.30 & 4.12 \\
\hline 00:01:20 & 163 & 71.3 & 2.276 & 2.117 & 24.1 & 1.07 & 17.07 & 4.20 \\
\hline $00: 01: 30$ & 162 & 81.0 & 2.622 & 2.372 & 27.0 & 1.11 & 17.15 & 4.25 \\
\hline 00:01:40 & 161 & 88.5 & 2.648 & 2.355 & 26.8 & 1.13 & 17.46 & 3.96 \\
\hline $00: 01: 50$ & 157 & 74.5 & 2.239 & 2.034 & 23.1 & 1.10 & 17.38 & 3.98 \\
\hline $00: 02: 00$ & 156 & 59.5 & 1.756 & 1.561 & 17.7 & 1.13 & 17.49 & 3.92 \\
\hline \multirow{2}{*}{\multicolumn{9}{|c|}{ Test Stage - Exercise }} \\
\hline & & & & & & & & \\
\hline 00:00:1n & 155 & 76.1 & 2.218 & 1.996 & 22.7 & 1.11 & 17.52 & 3.85 \\
\hline $00: 00: 20$ & 177 & 82.5 & 2.427 & 2.510 & 28.5 & 1.00 & 17.06 & 3.94 \\
\hline $00: 00: 30$ & 186 & 126.8 & 3.118 & 3.979 & 45.2 & 0.78 & 17.11 & 3.28 \\
\hline $00: 00: 40$ & 191 & 144.8 & 3.569 & 4.364 & 49.6 & 0.82 & 17.20 & 3.30 \\
\hline $00: 00: 50$ & 193 & 146.3 & 3.833 & 4.423 & 50.3 & 0.86 & 17.09 & 3.54 \\
\hline 00:01:00 & 199 & 173.6 & 4.363 & 4.472 & 50.8 & 0.98 & 17.50 & 3.44 \\
\hline 00:01:10 & 195 & 118.5 & 3.516 & 3.339 & 37.9 & 1.05 & 17.20 & 4.00 \\
\hline \multicolumn{9}{|c|}{ Test Stage -Recovery } \\
\hline 00:00:10 & 174 & 135.8 & 4.539 & 4.130 & 46.9 & 1.10 & 16.98 & 4.43 \\
\hline $00: 00: 20$ & 189 & 118.4 & 3.939 & 3.278 & 37.2 & 1.20 & 17.22 & 4.43 \\
\hline $00: 00: 30$ & 185 & 123.7 & 4.032 & 3.158 & 35.9 & 1.28 & 17.48 & 4.34 \\
\hline $00: 00: 40$ & 182 & 111.8 & 3.504 & 2.624 & 29.8 & 1.33 & 17.72 & 4.16 \\
\hline $00: 00: 50$ & 179 & 110.2 & 3.437 & 2.502 & 28.4 & 1.37 & 17.75 & 4.18 \\
\hline 00:01:00 & 178 & 118.3 & 3.518 & 2.501 & 28.4 & 1.41 & 18.01 & 3.95 \\
\hline 00:01:10 & 176 & 113.2 & 3.256 & 2.352 & 26.7 & 1.38 & 18.07 & 3.81 \\
\hline $00: 01: 20$ & 174 & 96.8 & 2.814 & 2.089 & 23.7 & 1.34 & 17.95 & 3.87 \\
\hline $00: 01: 30$ & 171 & 105.5 & 3.065 & 2.349 & 26.7 & 1.30 & 17.90 & 3.84 \\
\hline
\end{tabular}


Date:

Metabolic Edit -

Time Sec

HH:MM

00:01:40

00:01:50

00:02:00

00:02:10

00:02:20

00:02:30

00:02:40

00:02:50

00:03:00

00:03:10

00:03:20

00:03:30

00:03:40

00:03:50

00:04:00

00:04:10

00:04:20

00:04:30

00:04:40

00:04:50

00:05:00

00:05:10

00:05:20

00:05:30

00:05:40

$\begin{array}{rrrrr}H R & V E(B T P S) & V C O 2 & \begin{array}{r}\text { VO2 } \\ \text { BPM }\end{array} & \begin{array}{r}\text { VO2/kg } \\ \mathrm{mL} / \mathrm{kg} / \mathrm{min}\end{array} \\ 169 & 99.2 & 2.719 & 2.077 & 23.6 \\ 167 & 92.1 & 2.495 & 1.956 & 22.2 \\ 164 & 74.9 & 2.161 & 1.753 & 19.9 \\ 161 & 84.9 & 2.289 & 1.887 & 21.4 \\ 159 & 89.2 & 2.405 & 1.939 & 22.0 \\ 158 & 88.2 & 2.240 & 1.780 & 20.2 \\ 157 & 84.8 & 2.147 & 1.744 & 19.8 \\ 155 & 79.2 & 2.054 & 1.715 & 19.5 \\ 151 & 65.0 & 1.739 & 1.536 & 17.4 \\ 151 & 70.7 & 1.870 & 1.667 & 18.9 \\ 150 & 73.1 & 1.914 & 1.690 & 19.2 \\ 149 & 76.5 & 1.933 & 1.721 & 19.6 \\ 147 & 72.5 & 1.814 & 1.578 & 17.9 \\ 147 & 68.0 & 1.708 & 1.527 & 17.3 \\ 147 & 74.0 & 1.813 & 1.637 & 18.6 \\ 147 & 67.8 & 1.731 & 1.581 & 18.0 \\ 147 & 65.9 & 1.641 & 1.543 & 17.5 \\ 148 & 71.9 & 1.753 & 1.626 & 18.5 \\ 148 & 61.7 & 1.523 & 1.443 & 16.4 \\ 147 & 68.2 & 1.666 & 1.511 & 17.2 \\ 147 & 70.7 & 1.713 & 1.568 & 17.8 \\ 147 & 60.1 & 1.415 & 1.306 & 14.8 \\ 148 & 62.7 & 1.468 & 1.330 & 15.1 \\ 148 & 62.7 & 1.468 & 1.330 & 15.1 \\ 143 & 2.5 & 0.028 & 0.024 & 0.3\end{array}$

$R Q$
1.31
1.27
1.23
1.21
1.23
1.25
1.23
1.19
1.13
1.11
1.13
1.12
1.15
1.12
1.11
1.09
1.06
1.08
1.04
1.10
1.09
1.08
1.10
1.10
1.20

FEO2
$\%$
18.09
18.05
17.78
17.94
18.00
18.19
18.19
18.02
17.81
17.80
17.88
17.99
18.06
17.97
18.04
17.86
17.86
17.98
17.90
18.02
18.03
18.10
18.17
18.17
19.68

FECO2

$\%$
3.63

3.59

3.82

3.59

3.58

3.38

3.34

3.45

3.53

3.50

3.46

3.34

3.31

3.33

3.24

3.40

3.32

3.22

3.22

3.23

3.20

3.12

3.11

3.11

1.50 
Appendix L Borg Scale of Rating of Perceived Exertion 


\section{Rating of Perceived Exertion}

Very, very light

8

9

Very light

10

11

Fairly light

12

13

Somewhat hard

14

15

Hard

16

17

Very hard

18

19

Very, very hard

20 
Appendix M Blood Lactate Analysis Print-Out 
LACE Elack 8.5 moly

Tue 21/4/98 10:37:25

YSI 2S툐

LAC Elack 11.6 mol/L

Tue 21/4/98 10:39:57

YSI 2360

LAC Elack 6.6 mol L

Tue 21/4/98 16:44:14

YSI 23日0 